\title{
Selloffs, Bailouts, and Feedback: Can Asset Markets Inform Policy?*
}

\author{
RAPhael Boleslavsky David L. Kelly CURTIS R. TAYloR
}

FEBRUARY 1, 2017

\begin{abstract}
We introduce a new market microstructure model to study a setting in which an authority (e.g. a firm manager or government policymaker) learns about the likelihood of a bad state by observing activity in the asset market, before deciding whether to undertake a costly intervention to improve the state. Intervention erodes the value of an investor's private information by weakening the link between the initial state and the asset payoff. Informed investors are reluctant to make large, informative trades in the bad state, undermining the market's informativeness and the welfare gains generated by the possibility of a corrective intervention. Fundamentally, the authority faces a tradeoff between eliciting information from the asset market and using the information so obtained. The authority can generate a Pareto improvement if she commits to intervene less often when the market suggests that intervention is most beneficial and more often when the market suggests that intervention is unwarranted. She thus may benefit from imperfections in the intervention process or from delegating the decision to intervene to a biased agent.
\end{abstract}

JEL: C72, D82, G01, G14. market microstructure, corrective intervention, strategic trade

*Boleslavsky: r.boleslavsky@miami.edu, Kelly: dkelly@miami.edu, Taylor: crtaylor@duke.edu (corresponding).

(C) 2017. This manuscript version is made available under the Elsevier user license http://www.elsevier.com/open-access/userlicense/1.0/ 


\section{INTRODUCTION}

Asset markets provide investors with opportunities to profit from private information about fundamentals. The trading process, however, reveals some of the investors' information to other market participants and outside observers. Thus, activity in asset markets is a natural source of information about underlying conditions. Since the pioneering work of Mitchell and Burns (1938), economists have been aware of the power of asset markets to forecast business cycle fluctuations. In their influential article, Stock and Watson (2003) also found evidence that asset prices predicted inflation and output growth. Arrow et al. (2008) argue that markets' ability to convey information about asset fundamentals can be a valuable forecasting tool with "virtually limitless" applications (pg. 887). The idea that securities prices convey information is, of course, a central tenet of the efficient markets hypothesis (Malkiel and Fama 1970). Indeed, it is taken as common wisdom that asset prices augur the health of individual firms, industries, and even the entire economy.

This notion is not merely academic: markets influence both government and corporate policy. The TARP special inspector general's report explicitly cites asset market activity as a justification for offering Citibank "extraordinary assistance" (SIGTARP 2011). The regulatory case for using stock markets to assess the health of financial firms is documented by Curry, Elmer and Fissel (2003, 2007), Evanoff and Wall (2002), and Flannery (1998). The market's role in informing firms' mergers and acquisitions is analyzed by Kau, Linck and Rubin (2008) and Luo (2005), who conclude that merging companies do, indeed, rely on market activity to guide their decisions. A similar conclusion is drawn by Chen, Goldstein and Jiang (2007), who study the link between corporate investment, stock prices, and measures of price informativeness. Arrow et al. (2008) and Cowgill and Zitzewitz (2015) describe firms' use of internal prediction markets to inform decision processes. ${ }^{1}$

However, using activity in asset markets to inform managerial or public policy may be problematic. If (as in the model presented below) trade in the asset market is driven by a strategic, privately-informed investor (for example, a large hedge fund or investment bank) who is aware that a selloff could trigger a corrective intervention which improves the state, then the investor will have less incentive to sell the asset in the first place, and selloffs will be smaller and less informative. In other words, relying on market activity to guide corrective intervention can change the strategic investor's incentives, undermining the informational content of the very market in question.

To study this dilemma, we construct and analyze a new market microstructure model, wherein a privately informed investor anticipates that his trade in an Arrow security may trigger a corrective intervention by an "authority" - a firm manager or government policymaker — which changes the unobserved underlying state from bad to good at some cost. The authority relies on trade in the asset market to guide her decision: because intervention is costly, the authority wants to intervene only if she is sufficiently convinced that the state is likely to be bad, and she learns about the state by observing order flow or the market price. If the cost of intervention is small, then the authority

\footnotetext{
${ }^{1}$ The Wall Street Journal describes an incident in which Best Buy used an internal prediction market to assess the quality of a service line. When the service line was revealed to be weak by the prediction market, Best Buy redesigned the product.
} 
intervenes in equilibrium following all sell orders. Otherwise, the model admits a unique Perfect Bayesian equilibrium with some distinctive features. The authority never intervenes for small selloffs and intervenes randomly and with increasing probability for large ones. This generates a non-monotonicity in the equilibrium asset price: initially it falls with the magnitude of a selloff as the market becomes more convinced that the bad state will obtain and then it rises as the market anticipates the increasing likelihood of a corrective intervention. Compared with a benchmark setting in which interventions are infeasible, the expected value of the asset price is, therefore, higher. Also, because informed investors are discouraged from selling the asset, order flow is less (Blackwell) informative about the state. This effect reduces the authority's welfare gains from intervention - we show that the authority can generate a Pareto improvement by committing to limit intervention when it appears to be beneficial and triggering intervention when it appears to be unwarranted. The authority can approximate the commitment outcome by limiting its ability to intervene or by delegating the intervention decision to a biased agent.

These results arise because a corrective intervention erodes the value of the trader's private information. $^{2}$ If the intervention transitions the fundamental from the bad state to the good state (even stochastically), then the initial fundamental — the trader's private information - is less informative about the asset's final payoff. To highlight this effect most clearly, we focus primarily on an intervention that is perfectly effective, guaranteeing that the state is good whenever it is deployed. In this case, an intervention completely severs the link between the asset price and the trader's private information, rendering it valueless. ${ }^{3}$ The informed investor therefore adjusts his trading strategy to avoid triggering an intervention, selling less aggressively in the bad state.

We also consider an extension in which the authority observes only the asset price and - in particular - cannot base her intervention decision on the order flow. In this setting, the market price both anticipates the authority's intervention and-because the order flow is unobserved - directly informs it. We show that both of these roles cannot be fulfilled by a price that is purely a deterministic function of the order flow. In equilibrium the price is a deterministic, decreasing function of order flow for all buy orders and a range of small sell orders, but for large sell orders, the market maker sets the price stochastically, randomizing between the maximum equilibrium price (which is strictly larger than any deterministic price) and the minimum equilibrium price (the smallest deterministic price). Indeed, the price realization fully transmits the market maker's information about the state to the authority, who intervenes if and only if she observes the maximum price. In essence, the decision to intervene shifts to the market maker, who signals the authority to deploy an intervention by setting the maximum price. In fact, the unique equilibrium in this environment is payoff equivalent to the one where the authority directly observes order flow. Furthermore, for each order flow, the probability that the market maker induces an intervention is identical to the probability that the authority intervenes in the equilibrium with observable order flow, and the

\footnotetext{
${ }^{2}$ The intervention need not be corrective for this effect to arise. We focus on the case of corrective intervention, because it is most relevant in applications.

${ }^{3}$ In Section 7 we consider the possibility that the intervention sometimes fails to transition the state from bad to good, and demonstrate that committing to use an imperfect intervention process often benefits the authority.
} 
trader's equilibrium strategy is identical in the two settings.

Our analysis also delivers a sharp normative result: in equilibrium, she never benefits from the ability to intervene (regardless of whether the authority observes the order flow or price). It is sequentially rational to intervene whenever the authority believes that the state is sufficiently likely to be bad. However, to avoid the erosion of his private information caused by intervention, in equilibrium the investor adopts a less aggressive trading strategy that truncates beliefs at her point of indifference. Consequently, interventions that are strictly beneficial never take place in equilibrium. In other words, the erosion of private information that accompanies intervention also completely undermines the possible gains arising from the authority's ability to improve the state.

This irrelevance result leads us to investigate an ideal full-commitment setting in which the authority acts as a principal, designing an intervention policy that generates an ex ante Pareto improvement over the equilibrium that arises without commitment. The authority elicits more information from the market by committing to act in ways that are not sequentially rational. In particular, the ex ante optimal intervention policy is a clockwise rotation of the equilibrium policy, involving a larger probability of intervening following small selloffs, and a smaller probability of intervening following large ones. This insight finally motivates our investigation of two institutional remedies that are more realistic than the finely tuned ideal policy, but approximate it, namely the implementation of an imperfect intervention technology and delegation to a biased agent. ${ }^{4}$

An important component of our analysis is the strategic interaction between the trader and the authority. For this strategic interaction to occur, the trader must be large enough to move the market with his orders. In addition, the authority should expect that the trader may have access to relevant private information which underlies his market activity. These two attributes are complementary. Because of his ability to trade large quantities of the asset, private information is most valuable to a large investor. Thus, a large investor is most likely to acquire information that is sufficiently important to influence a policymaker or manager's intervention decision. A large investor is also likely to act strategically, anticipating the authority's response to his trades. Indeed, there is substantial evidence that the transactions of large traders, institutions, or insiders are based on superior information, and have significant impact on stock prices (Hendershott, Livdan and Schürhoff 2015, Chakravarty 2001, Ke, Huddart and Petroni 2003). Of particular relevance is Chan and Lakonishok (1995), who find that the impact of institutional buying on prices is larger than the impact of selling, corroborating a prediction of our analysis.

In the next section we discuss related literature. In Section 3 we present the model. In Section 4 we analyze a benchmark setting where interventions are infeasible. In Section 5 we characterize and analyze the unique Perfect Bayesian equilibrium of the game with interventions. In Section 6 we study two variants of the basic model, one in which the authority only observes the asset price and one in which the large informed investor competes with a group of small informed traders. In Section 7 we analyze the game assuming that the authority has commitment power. We also

\footnotetext{
${ }^{4}$ In an earlier draft we also showed that the authority could benefit from being non-transparent about her privatelyknown costs or benefits of intervening.
} 
analyze two cruder but more realistic forms of commitment. Section 8 concludes. Proofs and lemmas are in the appendix.

\section{RELATED LITERATURE}

From a methodological perspective, we introduce a new market microstructure model that incorporates continuously distributed noise trades and interventions into the single-arrival frameworks of Glosten and Milgrom (1985) and Easley and O'Hara (1987). Unlike standard settings in which noise trades - and consequently informed trades - are discrete, in our model the noise trader's order flow is distributed continuously, which induces informed traders to also mix continuously in equilibrium. Hence, the equilibrium order flow in our single-arrival model is continuously distributed, with more extreme orders conveying more information (as in the batch-order model of Kyle (1985)). By avoiding discontinuities arising from discrete changes in the trader's equilibrium strategy, our analysis generates an equilibrium that is continuous in the model parameters, enhancing tractability. In particular, our model has only two equilibrium cases (with a continuous transition between them) and one case of primary interest. ${ }^{5}$ In addition, the equilibrium is unique (in the main case of interest) and exhibits intuitive comparative statics. We believe that the tractability and parsimony of our model may prove useful in a variety of settings beyond the one we study here.

From an applied perspective, our paper contributes to the burgeoning literature on feedback between firm or government policy and asset prices. ${ }^{6}$ In order to clarify our specific contributions, we now discuss our findings in the context of the four papers in the feedback literature that are most closely related to ours.

First, Dow, Goldstein and Guembel (2008) consider incentives for information acquisition in a market with a continuum of infinitesimal speculators. The focus of their paper is on the connection between the market's prior beliefs with incentives for information acquisition, showing that when the prior information is good, more information is acquired and imputed into prices than when the prior belief is bad. Thus, the main mechanism underlying the analysis operates through (infinitesimal) traders' incentives to acquire information about the state. In contrast, our analysis deliberately abstracts away from issues related to information acquisition, showing that the possibility of corrective intervention may impede the market's ability to impute information that traders already possess or can acquire at no cost.

Second, Bond, Goldstein and Prescott (2010) consider feedback between an asset price and a manager's corrective action, treating the market as a rational expectations pricing function. The main result of their paper is the non-monotonicity of the price as a function of the fundamental, which obscures the manager's ability to learn from the price. Unfortunately, rational expectations equilibria may fail to exist in their setting. In our model, the equilibrium asset price is also

\footnotetext{
${ }^{5}$ For example, the analysis of Edmans, Goldstein and Jiang (2014) (discussed below), considers feedback between markets and real decisions in a discrete model with numerous cases.

${ }^{6}$ See for example, Dow and Gorton (1997), Subrahmanyam and Titman (1999), Angeletos, Lorenzoni and Pavan (2010), Fulghieria and Lukin (2011).
} 
non-monotonic in the size of the investor's sell order, but this non-monotonicity does not obscure information. In our baseline model, information about the fundamental is conveyed by the magnitude of the order, flow, which the authority observes directly. As noted in the Introduction, however, we also study a version of our model in which the authority observes only the asset price. Remarkably - even in this setting - all information about the state contained in the order is fully transmitted. Specifically, while the expected equilibrium asset price is still non-monotonic, the price itself depends on the order flow stochastically, and the authority correctly infers that she should intervene for high price realizations and refrain from intervening for low ones. Moreover, this is the unique equilibrium in this version of the model and it always exists. To our knowledge, this is the first instance in this literature of a random equilibrium asset price.

Third, Bond and Goldstein (2014) consider government interventions in a Grossman and Stiglitz (1980) market framework. In their analysis, the level of financial support that the government would like to offer depends on an attribute of the firm (for example the social surplus generated by its activity), about which the government is uncertain. Information about this attribute is dispersed among risk averse investors, and it is aggregated into the market price. Thus, the government infers information about the firm's attribute from the price when deciding how much support to offer. However, because the firm's cash flow and the value of the asset being traded depend on the government's transfer to the firm, the investors anticipate the government's intervention when trading the asset.

Our investigation differs from Bond and Goldstein (2014) in several key respects. First, we focus on the incentives of a large strategic investor to suppress information transmission through his trading strategy. ${ }^{7}$ Second, we show that the authority learns valuable information only if she can commit not to intervene too frequently and we suggest two institutional methods for securing commitment power. Finally, the central mechanisms underlying the feedback between trade and intervention are different in our analysis. In Bond and Goldstein (2014), traders are risk averse; the transfer policy used by the government changes the risk profile of the asset and can either stimulate or dampen the investors' incentives to trade. In contrast, in our model corrective intervention erodes the value of private information and always dampens the informed investor's incentive to take a short position in the asset.

Finally and perhaps most relevantly, Edmans, Goldstein and Jiang (2014) study a firm's decision to abandon or expand an investment in response to trade in a binary version of the Kyle (1985) model, with two states, two trade sizes, a noise trader and an informed investor (who exists with probability less than one), and an exogenous transaction cost for market participation. Edmans, Goldstein and Jiang (2014) show that if transaction costs faced by the informed investor are not too large and not too small, then an equilibrium exists in which the informed investor buys one unit if

\footnotetext{
${ }^{7}$ Our analysis of a large strategic trader is also a contribution relative to Dow, Goldstein and Guembel (2008), who consider infinitesimal traders, and Bond, Goldstein and Prescott (2010), who treat the market as a reduced form rational expectation pricing function. See also Dow and Gorton (1997), Subrahmanyam and Titman (1999), and Goldstein, Ozdenoren and Yuan (2011), where policymakers obtain useful information because traders are infinitesmal or have aligned interests with the policymaker.
} 
he knows that the state is good, but abandons the market if he knows that the state is bad (referred to as the "buy, not sell" equilibrium). Because only the noise trader sells in this equilibrium, large selloffs, involving two rather than one share, never take place on the equilibrium path and every sale (of one unit) triggers an intervention with certainty. In order to reconcile this equilibrium with real-world examples of large selloffs leading to corrective action, the authors suppose that the actual corrective action must have been unanticipated by the informed investors. ${ }^{8}$

The unique equilibrium in our model features less-extreme (and arguably more realistic) behavior by both the informed trader and the authority who controls the intervention. In our equilibrium, the informed trader participates in the market in the bad state, even though he anticipates that his trade may trigger a corrective intervention. He responds to the possibility of intervention by selling less aggressively, shifting probability mass from larger to smaller orders, but he does not abandon the market altogether. Our authority is also less reactive. In the equilibrium of Edmans, Goldstein and Jiang (2014), observed selloffs are always small, involving one (never two) shares, and any selloff always triggers an intervention. In contrast, in our analysis small selloffs do not trigger interventions by the authority while larger selloffs are increasingly likely to do so (in a continuous way). Because of these features, our characterization is easier to reconcile with intuition and with actual cases in which large selloffs have led to corrective interventions without the need to assume that traders are surprised. To be clear, the main equilibrium of Edmans, Goldstein and Jiang (2014) predicts that small selloffs lead to interventions (for certain), while our main equilibrium predicts that small selloffs do not lead to intervention and larger observed selloffs are increasingly likely to do so.

Furthermore, while Edmans, Goldstein and Jiang (2014) is primarily a positive analysis, our analysis also incorporates normative issues related to intervention policy. As noted above, we make two novel contributions in this dimension: we characterize the Pareto optimal intervention policy under full commitment by the authority and investigate two alternative institutions that approximate the ideal mechanism. This aspect of our contribution has no analogue in Edmans, Goldstein and Jiang (2014).

Our paper also touches on the recent theoretical literature on government bailouts of financial entities (Philippon and Skreta 2010, Farhi and Tirole 2012, Tirole 2012). Relative to these papers, we take a more agnostic approach both on the need for and implementation of a bailout (a corrective intervention) and focus on a somewhat different set of questions. Rather than the design of an optimal bailout mechanism, our analysis is concerned with how an authority can extract information from asset markets to learn about the firm's fundamental and to determine whether a bailout is needed. In this respect, our paper also relates to the literature on information aggregation in prediction markets (Wolfers and Zitzewitz 2004, Arrow et al. 2008, Cowgill, Wolfers and Zitzewitz 2009, Snowberg, Wolfers and Zitzewitz 2012), which are commonly viewed as efficient institutions for eliciting information. Our results suggest a caveat: prediction markets are informative provided

\footnotetext{
${ }^{8}$ See footnote 1 of Edmans, Goldstein and Jiang (2014) and their discussion of Coca-Cola's attempted acquisition of Quaker Oats on page 3767.
} 
that decision-makers (who watch the market in order to learn about the state) cannot take actions that affect the state; otherwise, informativeness may be compromised. ${ }^{9}$

There is also a literature that studies a setting in which information flows in the opposite direction from the one we consider (for example, Angeletos, Hellwig and Pavan 2006, Baeriswyl and Cornand 2010, Frankel and Kartik 2016). In these papers the authority's actions convey information to market participants rather than the other way round. Regardless of the direction of the information flow, it turns out that the market's ability to convey private information is limited. In our setting, a strategic trader limits trade and market informativeness to prevent the authority from using the information in a way which reduces trading profits. In the opposite-flow setting, the authority alters its actions in order to manipulate the beliefs of market participants, reducing the market's information efficiency.

Another related literature studies the interaction between information disclosure policies and market prices. Amador and Weill (2010), show that better information from the central bank may reduce the informativeness of the price system, reducing overall economic efficiency. Kurlat and Veldkamp (2015) show that forcing asset issuers to disclose payoff relevant information can improve economic efficiency, but it also makes returns less uncertain, thereby reducing investors' compensation for taking on risk, which may leave them worse off. Finally, our paper relates to a literature on trade in which the object's value depends on market participants' subsequent actions, which are themselves informed by market activity (Bond and Eraslan 2010, Atakan and Ekmekci 2014). In our setting, asset value is influenced by the intervention of a third-party.

\section{THE MODEL}

We study a game with two active risk-neutral players, an investor and an authority (e.g. a firm manager or government policymaker), and a passive market maker who takes the other side of any trade with the investor. Two states of nature are possible: $\omega=0$, which occurs with prior probability $q$, and $\omega=1$, which occurs with $1-q$. The investor may buy or sell shares of an Arrow security that commits the seller to pay the buyer one if $\omega=1$ and zero if $\omega=0 .{ }^{10}$ The asset price is equal to its expected payoff given all publicly available information, and it adjusts instantly to arrival of new information. Investors can submit either buy or sell orders. Because interventions correct the state from zero to one, they happen in response to sell orders; therefore, the focus of the paper is primarily on the sell side of the market. Consequently, it is notationally convenient to adopt the convention that a positive order represents a sale and a negative order represents a purchase. We often refer to the size of a trader's order, which is the order's absolute value.

The investor is an informed trader with probability $a$ and a noise trader with probability $1-a$. An informed trader privately observes a signal realization (his type) $i \in\{0,1\}$ which is perfectly correlated with $\omega$, and invests in an effort to maximize his expected return. A noise trader invests

\footnotetext{
${ }^{9}$ Other issues with prediction markets are addressed by Ottaviani and Sørensen (2007) and Hanson and Oprea (2009), who analyze traders' incentives to manipulate the underlying state or market price.

${ }^{10}$ It is formally equivalent to suppose that the asset has "fundamental" value $\omega$ in state $\omega$ and allow short sales.
} 
for exogenous reasons (e.g., a liquidity shock) and generates a random order flow. We represent the noise trader's order flow as the realization of a compound lottery. The first realization determines the sign of the trader's order, i.e. whether the noise trader sells or buys. With probability $s \in(0,1)$ the noise trader submits a (positive) sell order, and with probability $1-s$, the noise trader submits a (negative) buy order. The second realization determines the size of the order: if the order is a sell, then it is drawn from continuously differentiable density $f_{s}(\cdot)$ with full support on $[0, \infty)$. If the order is a buy, then it is drawn from continuously differentiable density $f_{b}(\cdot)$ with full support on $(-\infty, 0]$. We denote the associated cumulative distribution functions by $F_{s}(\cdot)$ and $F_{b}(\cdot)$ for sells and buys respectively. We also assume that the expected values of the noise trader's buy and sell orders are finite. ${ }^{11}$

The authority cares intrinsically about the state. In particular, she receives payoffs normalized to one if $\omega=1$ and zero if $\omega=0$. The authority has a costly technology that allows her to "intervene" in the process that generates the state. If she intervenes, then she bears cost $c<1$ but guarantees that the state is $\omega=1$ with probability one. ${ }^{12}$ We focus on the case in which the intervention is sufficiently costly that the authority would not want to intervene under the prior, $c>q$. She may, however, find it optimal to intervene if the investor's trade (e.g. a large sell order) reveals that the state is likely to be $\omega=0 .{ }^{13}$

The game proceeds in four stages. In the first stage the state and the trader's type (zero, one or noise) are realized. In the second stage the trader submits an order $t \in \Re$ to the market, observed publicly. The market maker updates his beliefs based on the order and adjusts the price to equal the asset's expected value and then fills the order. ${ }^{14}$ In the third stage, the authority observes the trade and decides whether to intervene. In the last stage the (terminal) state is revealed and all payoffs are realized.

Notation. A mixed strategy for the type $i \in\{0,1\}$ trader is a probability measure over $t \in \Re$, represented by cumulative distribution function $\Phi_{i}(\cdot)$. We allow $\Phi_{i}(\cdot)$ to have jump discontinuities, thereby allowing for mass points. ${ }^{15}$ We denote the associated density by $\phi_{i}(t)$, for all $t \in \Re$ at which the distribution function is differentiable. The equilibrium order flow, denoted by $\tau$, is realized from $\Phi_{0}(\cdot)$ with probability $a q$, from $F_{s}(\cdot)$ with probability $(1-a) s$, from $\Phi_{1}(\cdot)$ with probability $a(1-q)$, and from $F_{b}(\cdot)$ with probability $(1-a)(1-s)$. Let $\chi(t)$ be the authority and the market maker's belief that the state is zero conditional on observing order $t \in \Re: \chi(t) \equiv \operatorname{Pr}(\omega=0 \mid \tau=t)$, and let $\alpha(t)$ be the probability that the authority intervenes after observing order flow $t \in \Re$.

\footnotetext{
${ }^{11}$ The assumption that the support of the noise trade distribution is unbounded is standard (Kyle 1985). Qualitatively identical results for bounded distributions are available from the authors upon request.

${ }^{12}$ In section 7 we consider a case in which the intervention does not succeed with certainty.

${ }^{13}$ As presented, the authority cannot learn the state before intervening. Allowing the authority to perform a costly audit which allows it to learn the state before intervening gives equivalent results (proof upon request).

${ }^{14}$ An equivalent assumption is that competing market makers post price schedules in stage one, assigning a price per share for each order size $t \in \Re$; in this way, the trader observes the price before submitting his order. Under Bertrand competition, market makers compete away all profits, and the price of the asset conditional on the order size is always equal to its expected worth. If the trader can only sell one share or buy one share, the difference between the price at which the market maker buys and the price at which the market maker sells is the bid-ask spread; see Glosten and Milgrom (1985) for additional discussion.

${ }^{15}$ This possibility is largely irrelevant: in our main case of interest mass points are ruled out in Lemma A.2.
} 
Equilibrium. The solution concept is Perfect Bayesian equilibrium, which consists of a trading strategy for each type of informed investor, $\left(\Phi_{0}(t), \Phi_{1}(t)\right)$, an intervention strategy for the authority, $\alpha(t)$, and a belief function $\chi(t)$ all on domain $\Re$. Each player's strategy must be sequentially rational given the strategy of the other players, and for each order $t \in \Re$ the belief function must be consistent with Bayes' rule applied to equilibrium strategies.

Market Price. The asset is worth one if $\omega=1$ and zero if $\omega=0$. Therefore, its price must equal the probability that $\omega=1$ at the end of the game, conditional on order $t \in \Re$ :

$$
p(t)=(1-\chi(t))(1-\alpha(t))+\alpha(t)=1-\chi(t)+\alpha(t) \chi(t) .
$$

If an intervention takes place, the asset is worth one for certain, but if the intervention does not take place, then the expected payoff of the asset is equal to $1-\chi(t)$, the probability that the state is one, given the observed order. The price therefore incorporates information about both the "fundamental" (decreasing in $\chi(t)$ ) and about the anticipated intervention policy (increasing in $\alpha(t))$.

Trader Payoffs. Suppose that the type-0 trader submits a sell order, $t>0$. He collects the sale price, $p(t)$ on each share sold. In the absence of intervention, he knows that $\omega=0$, and he will not owe any money to the market maker. If intervention occurs, however, he will owe one per share sold. Therefore, on each share that he sells the type-0 trader expects profit $p(t)-\alpha(t)$. Conversely, if he submits a buy order, $t<0$, then he expects $\alpha(t)-p(t)$ per share. Combining, we find that a type-0 trader's expected payoff from submitting order $t \in \Re$ is

$$
\begin{aligned}
u_{0}(t) & =t(p(t)-\alpha(t)) \\
& =t(1-\chi(t))(1-\alpha(t)) .
\end{aligned}
$$

The type-1 trader expects the asset to be worth one whether or not an intervention takes place. Thus, if the type- 1 trader submits buy order $t<0$, then he expects profit $1-p(t)$ on each share bought, and if he submits a sell order, then he expects profit $p(t)-1$ on each share sold. A type-1 trader's expected payoff from submitting order $t \in \Re$ is thus

$$
\begin{aligned}
u_{1}(t) & =-t(1-p(t)) \\
& =-t \chi(t)(1-\alpha(t)) .
\end{aligned}
$$

To understand the structure of payoffs in $(2,3)$, observe that in the absence of interventions $(\alpha(t)=$ 0 ), the type-0 (type-1) informed trader expects a positive rent from selling (buying), unless the order fully reveals his private information. Indeed, whenever the market maker is uncertain about the true state, he sells the asset too cheaply to a type-1 trader $(p(t)<1)$, and he buys it too expensively from a type- 0 trader $(p(t)>0)$. The possibility of intervention does not change the mis-pricing that arises from asymmetric information. However, conditional on an intervention taking place, the state is known to be $\omega=1$; asymmetric information vanishes, and with it, the trader's rent. 
Thus the trader's expected payoff when interventions occur with positive probability is simply his expected payoff in the absence of interventions, multiplied by the probability that no intervention takes place. Consequently, (holding beliefs constant) increasing the probability of intervention for a particular order reduces the profit that the order generates.

Weak Dominance. Equations (2) and (3) immediately give the following result.

Lemma 3.1 (Weak dominance). Given any authority strategy $\alpha(\cdot)$, submitting any buy (sell) order delivers the type-0 (type-1) trader a weakly smaller payoff than placing no order:

$$
t<0 \Rightarrow u_{0}(t) \leq u_{0}(0) \text { and } \quad t>0 \Rightarrow u_{1}(t) \leq u_{1}(0)
$$

In light of this lemma, we focus on equilibria in which the type- 0 trader only submits sell orders and the type- 1 trader only submits buy orders.

Authority Payoff. The authority's expected payoff from intervening with probability $\alpha$ is

$$
(1-\alpha)(1-\chi(t))+\alpha(1-c)=1-\chi(t)+\alpha(\chi(t)-c)
$$

If the authority intervenes, she ensures $\omega=1$, but loses $c$; if she does not intervene, then she receives payoff one whenever $\omega=1$. Hence, the authority's equilibrium intervention strategy must satisfy the following sequential rationality condition:

$$
\alpha(t)=\left\{\begin{array}{ccc}
0 & \text { if } & \chi(t)<c \\
{[0,1]} & \text { if } & \chi(t)=c \\
1 & \text { if } & \chi(t)>c
\end{array}\right.
$$

Thus, the authority intervenes whenever the probability that the state is low exceeds the intervention cost. This condition highlights the dilemma facing an informed trader. If he executes a trade $t$ that reveals too much information, so that $\chi(t)>c$, then the market maker will anticipate an intervention and will set $p(t)=1$, resulting in a payoff of zero for the trader. The investor must therefore be cognizant of precisely how his trades impact beliefs.

Equilibrium Beliefs. In equilibrium, beliefs are determined by Bayes' Rule applied to strategies. A buy order either comes from a type- 1 informed trader or a noise trader. Thus, for $t<0$ at which $\phi_{1}(t)$ exists,

$$
\chi(t)=\frac{q(1-a)(1-s) f_{b}(t)}{(1-q) a \phi_{1}(t)+(1-a)(1-s) f_{b}(t)},
$$

and $\chi(t)=0$ if $t<0$ is a mass point. Likewise, a sell order either comes from a type- 0 informed trader or a noise trader. Thus, for $t>0$ at which $\phi_{0}(t)$ exists,

$$
\chi(t)=\frac{q\left(a \phi_{0}(t)+(1-a) s f_{s}(t)\right)}{q a \phi_{0}(t)+(1-a) s f_{s}(t)},
$$


and $\chi(t)=1$ if $t>0$ is a mass point. Note that if an informed investor increases the probability mass on a particular order, then the order reveals more of the investor's private information: for buy orders $(t<0), \chi(t)$ is decreasing in $\phi_{1}(t)$, and for sell orders $(t>0), \chi(t)$ is increasing in $\phi_{0}(t)$.

Additional Notation. For ease of exposition, we define the following transformations of the parameters of the model:

$$
\begin{aligned}
J(c, q) & \equiv \frac{1-q}{1-c} \quad(c, q) \in[0,1]^{2} \\
K_{i}(a, q, s) & \equiv \frac{a}{1-a}\left((1-i) \frac{q}{s}+i \frac{1-q}{1-s}\right) \quad(a, q, s) \in[0,1]^{3} \text { and } i \in\{0,1\} .
\end{aligned}
$$

Throughout we suppress arguments $(c, q)$ and $(a, q, s)$. Parameters $\left(K_{0}, K_{1}\right)$ are measures of market informativeness. To see this, note that $a q$ is the prior probability that a sell order originates with the informed trader, while $(1-a) s$ is the prior probability that a sell order originates with the noise trader. Therefore, parameter $K_{0}$ is the likelihood ratio (under the prior) that a sell order originates with the type- 0 informed trader. Likewise, $K_{1}$ is the likelihood ratio that a buy order originates with the type-1 informed trader. Holding the informed investor's trading strategy constant, an increase in either of these parameters moves beliefs closer to the true state, i.e. $\chi(t)$ is increasing in $K_{0}$ for sell orders and decreasing in $K_{1}$ for buy orders. To interpret $J$, recall that the authority prefers not to intervene under the prior belief. Parameter $J>1$ measures the intensity of this preference: it is the ratio of the authority's payoffs without intervention $(1-q)$ and with intervention $(1-c)$.

Equilibrium Structure. A number of observations follow from some preliminary analysis. Lemma A.1 (in the appendix) establishes that $\chi(t) \leq q$ following any buy order and $\chi(t) \geq q$ for any sell order. Consequently, $\alpha(t)=0$ for any buy order in equilibrium (consult (4)). Lemma A.2 (also in the appendix) establishes that in any equilibrium in which the traders expect positive profit, both types of trader play mixed strategies with no gaps or mass points. ${ }^{16}$ Furthermore, the support of the type- 0 trader's mixed strategy is an interval $\left[m_{0}, \infty\right)$ and type- 1 's is $\left(-\infty,-m_{1}\right]$, for some $m_{0} \geq 0$ and $m_{1} \geq 0$. In order to avoid revealing his private information to the market maker (or authority) the informed trader must "hide in the noise" generated by the noise trader's order flow. Moreover, the type- 0 trader's equilibrium payoff is equal to $m_{0}(1-q)$ and the type- 1 's is $m_{1} q$. Intuitively, because $m_{i}$ is the threshold value separating the set of orders which could only be submitted by the noise trader from the set of orders that could be submitted by either the noise or informed trader, $m_{i}$ is the largest size order that does not affect beliefs, generating payoffs $u_{0}=m_{0}(1-q)$ and $u_{1}=m_{1} q$ (consult $\left.(2,3)\right) .{ }^{17}$ Consequently, any other order in the support of the informed trader's mixing distribution must deliver the same payoff.

\footnotetext{
${ }^{16}$ If the type- 0 trader's equilibrium strategy has a mass point on order $t$, then $\chi(t)=1$ (consult $\left.(6)\right)$. If $\chi(t)=1$, then $u_{0}(t)=0$ (consult (2)). Thus, if the informed seller submits this order as part of his equilibrium mixed strategy, then his payoff must be zero.

${ }^{17}$ Note that because order $m_{i}$ does not affect beliefs and $c>q$, the probability of intervention following order $m_{i}$ must be zero.
} 


\section{NO INTERVENTIONS}

As a benchmark, we first present the case in which interventions are infeasible. In equilibrium, each type of informed trader must be indifferent over all trades inside the support of his mixed strategy. Using this observation allows us to determine the mixing densities, $\phi_{i}(\cdot)$, parameterized by the minimum trade size $m_{i}$. We then invoke Lemma A.2 which provides a connection between the trader's equilibrium payoff and minimum trade size. Finally, we solve for the equilibrium minimum trade size by ensuring that the trader's mixing density integrates to one (see the appendix for details). The following proposition characterizes the equilibrium and comparative statics.

Proposition 4.1 (No interventions). The unique equilibrium when interventions are not possible is characterized as follows. Let

$$
G_{s}^{*}(m) \equiv \int_{m}^{\infty} \frac{t-m}{K_{0} m} f_{s}(t) \mathrm{d} t \quad \text { and } \quad G_{b}^{*}(m) \equiv \int_{m}^{\infty} \frac{t-m}{K_{1} m} f_{b}(-t) \mathrm{d} t .
$$

Strategies. Type-0 plays a mixed strategy $\phi_{0}^{*}(t)$ on support $\left[m_{0}^{*}, \infty\right)$, and type-1 plays a mixed strategy $\phi_{1}^{*}(t)$ on support $\left(-\infty,-m_{1}^{*}\right]$, where

$$
\phi_{0}^{*}(t)=\frac{t-m_{0}^{*}}{K_{0} m_{0}^{*}} f_{s}(t) \text { and } \phi_{1}^{*}(t)=\frac{|t|-m_{1}^{*}}{K_{1} m_{1}^{*}} f_{b}(t)
$$

and $\left(m_{0}^{*}, m_{1}^{*}\right)$ are the unique values that satisfy $G_{s}^{*}\left(m_{0}^{*}\right)=1$ and $G_{b}^{*}\left(m_{1}^{*}\right)=1$. In addition, $m_{i}^{*}>0$.

Payoffs. Informed trader payoffs are $u_{1}=m_{1}^{*} q$ and $u_{0}=m_{0}^{*}(1-q)$.

Comparative Statics. (i) An increase in a causes $m_{i}^{*}$ to decrease, both types to trade less aggressively in the sense of monotone likelihood ratio, and a fall in type $i$ 's equilibrium expected payoff $u_{i}$.

(ii) An increase in $q$ causes an increase in $m_{1}^{*}$ and decrease in $m_{0}^{*}$, type 1 to trade more aggressively and type 0 to trade less aggressively in the sense of monotone likelihood ratio, a rise in type 1's equilibrium expected payoff $u_{1}$, and a fall in type 0's equilibrium expected payoff $u_{0}$. (iii) If the noise trader sells (buys) more aggressively in the sense of first order stochastic dominance, then $m_{0}^{*}$ and $u_{0}^{*}\left(m_{1}^{*}\right.$ and $\left.u_{1}^{*}\right)$ weakly increase. (iv) If the noise trader sells (buys) more aggressively in the sense of monotone likelihood ratio, then type-0 (type-1) sells (buys) more aggressively in the sense of monotone likelihood ratio.

From the expressions for the informed trader's payoffs (2) and (3)), it is clear that-holding beliefs fixed - an informed investor benefits by increasing the size of his order. In equilibrium, however, beliefs are not fixed. Indeed, precisely because large orders are tempting for the informed trader, they also reveal more information to the market maker, generating larger movements in beliefs and less favorable transaction prices. Because the investor must be indifferent over all trades in the support of his mixed strategy, in equilibrium the effect on the belief (and the price) must exactly offset the benefit of a larger trade size. In other words, large sell (buy) orders are associated with a relatively high (low) belief that $\omega=0$ and correspondingly low (high) asset price. 
The trader's payoff functions reveal the exact connection between equilibrium beliefs and order flow over the support of the trader's mixed strategy. In equilibrium,

$$
\begin{aligned}
\text { Buy order } t \in\left(-\infty,-m_{1}^{*}\right] & \Rightarrow \chi(t)=\frac{m_{1}^{*} q}{|t|} \\
\text { Order } t \in\left[-m_{1}^{*}, m_{0}^{*}\right] & \Rightarrow \chi(t)=q \\
\text { Sell order } t \in\left[m_{0}^{*}, \infty\right) & \Rightarrow \chi(t)=1-\frac{m_{0}^{*}(1-q)}{t} .
\end{aligned}
$$

Note that, in equilibrium no order perfectly reveals the state, but arbitrarily large orders are "nearly" perfectly revealing:

$$
\lim _{t \rightarrow \infty} \chi(t)=1 \text { and } \lim _{t \rightarrow-\infty} \chi(t)=0 .
$$

Therefore, the set of posterior beliefs $\chi(t)$ that arises with positive likelihood in equilibrium is the open interval $(0,1)$. Figure 1 illustrates the equilibrium beliefs as a function of order flow.

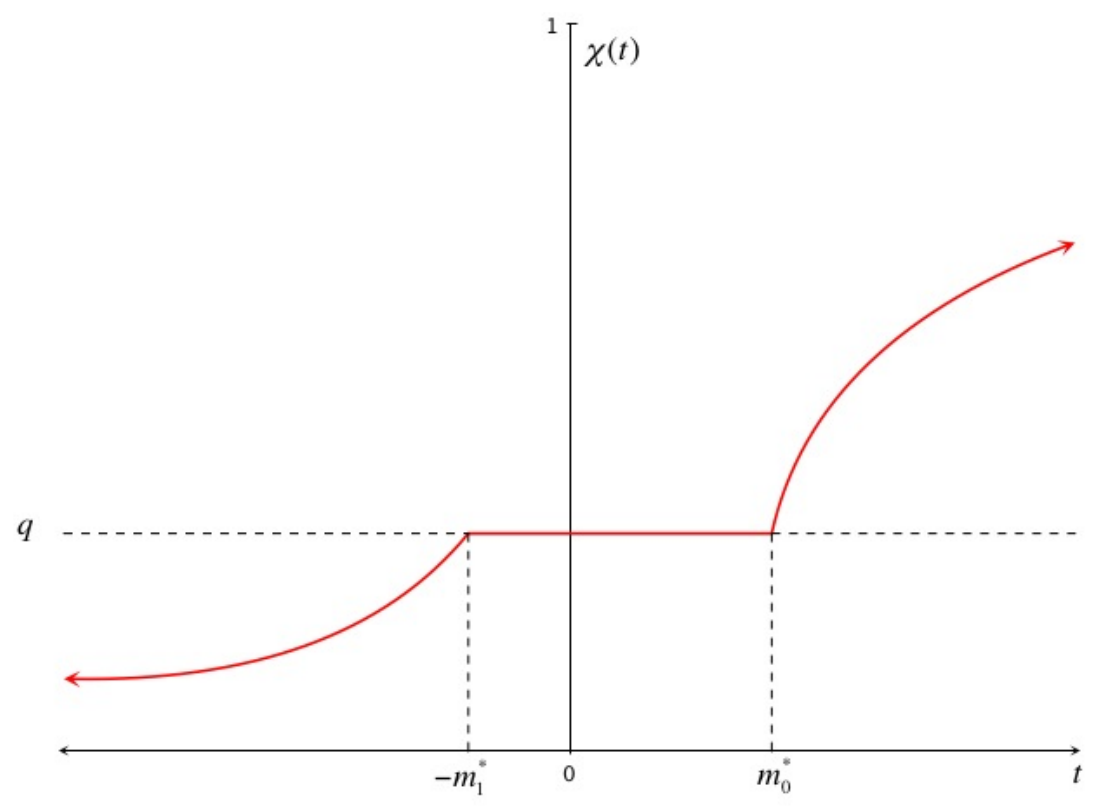

Figure 1: THE POSTERIOR BeLIEF WITH NO POSSIBILITY OF INTERVENTION

The comparative statics in Proposition 4.1 are natural. When the probability that the trader is informed, $a$, is high, the market maker's beliefs (and hence prices) are very sensitive to order flow. Both types of informed trader mix over a wide range of orders using a density that more closely approximates the noise trader's. Because the noise trader is unlikely to be present, this possibility provides weak "cover" for the informed trader, yielding meager information rents. By contrast, when $a$ is low, order flow is most likely generated by a noise trader and prices are, therefore, 
relatively insensitive. Hence, an informed investor can make large trades without causing large movements in the price, thereby securing substantial information rents. Similarly, when the prior is more biased toward state zero, i.e. $q$ is high, a sell order is relatively more likely to have come from an informed trader and a buy order is relatively more likely to have come from a noise trader. Thus, sell orders - which confirm the prior - generate large downward movements in the price and buy orders - which contradict the prior - generate small upward movements. Hence, an informed buyer can trade more aggressively without revealing his information and secure correspondingly higher rents than an informed seller. Of course, the reverse comparative statics (and intuition) hold when $1-q$ is high. Furthermore, as the noise trader becomes more aggressive, the informed trader also can be more aggressive without revealing too much private information about the state.

\section{INTERVENTIONS}

With the benchmark of the preceding section in hand, we now turn to a setting in which interventions are possible. First note that because $\chi(t) \leq q$ following buy orders, a buy order never leads to intervention. Hence, the possibility of intervention does not affect the type- 1 trader's equilibrium behavior and payoff, which are identical to the ones in Proposition 4.1. For this reason, we focus in what follows on the equilibrium strategies of the type-0 trader and the authority.

Imagine for a moment that when it is time for her to act, the authority observes that a buy order was not placed, but she has no other information about the order. Because type-1 investors always buy in equilibrium, this limited information reveals the trader to be either a noise trader (with probability $1-a$ ) or a type-0 (with probability $a$ ), indicating that the state is more likely to be $\omega=0$. In this scenario, Bayes' rule implies the following posterior belief that the state is zero is:

$$
\widehat{\chi} \equiv \frac{q(a+(1-a) s)}{q a+(1-a) s}=\frac{K_{0}+q}{K_{0}+1}>q .
$$

If $a$ is high enough or $c$ is low enough so that $c \leq \hat{\chi}$, then simply knowing that a buy order was not submitted is sufficient to induce the authority to intervene. In the following proposition, we show that in this case in every equilibrium the authority intervenes after observing any sell order, no matter how small, and the type-0 trader's equilibrium payoff is zero (consult (2)). We also show that in the opposite case the intervention probability is less than one following every sell order, which leaves type-0 with profitable trading opportunities and a strictly positive equilibrium payoff.

Proposition 5.1 (Low cost vs. High cost).

- If $c \leq \hat{\chi}$, then a multiplicity of equilibria exists. In every equilibrium, the type-0 trader's expected profit is zero and $\alpha(t)=1$ for all $t>0$.

- If $c>\hat{\chi}$, then the type-0 trader's equilibrium expected profit must be strictly positive and $\alpha(t)<1$ for all $t>0$. 
For the remainder of the paper, we focus on the case in which $c>\widehat{\chi}$, so that the authority does not intervene for certain following every possible sell order.

When $c>\hat{\chi}$, the possibility of intervention imposes a constraint on the information revealed by sell orders in equilibrium. According to Proposition 5.1, if $c>\hat{\chi}$, then the type-0 trader's equilibrium payoff must be strictly positive, and $\alpha(t)<1$ for each $t$ inside the support of the type- 0 trader's mixed strategy. Therefore, for all sell orders, $\chi(t) \leq c$ in equilibrium. This condition rules out mass points and yields an upper bound on the type- 0 trader's equilibrium mixing density:

$$
\chi(t) \leq c \Longleftrightarrow \phi_{0}(t) \leq \frac{J-1}{K_{0}} f_{s}(t) .
$$

In the equilibrium of the no intervention benchmark, arbitrarily large sell orders reveal nearly all information (consult (8)), violating condition (9). Therefore, the no intervention equilibrium breaks down when intervention is possible - that is, intervention occurs with strictly positive probability in equilibrium. Furthermore, if the authority intervenes with non-zero probability following order $t$, then condition (9) must hold with equality.

The following proposition characterizes the unique equilibrium in our main case of interest. It is derived in an analogous way to Proposition 4.1, imposing (9) as an additional condition.

Proposition 5.2 (Stochastic Interventions). When $c \in(\widehat{\chi}, 1)$ and interventions are possible, the game has a unique equilibrium, characterized as follows. Let

$$
G^{\dagger}(m) \equiv \int_{m}^{J m} \frac{t-m}{K_{0} m} f_{s}(t) \mathrm{d} t+\frac{J-1}{K_{0}}\left(1-F_{s}(J m)\right) .
$$

Strategies. The type-0 trader places a sell order distributed according to continuous probability density function $\phi_{0}^{\dagger}(t)$ over support $\left[m_{0}^{\dagger}, \infty\right)$ defined piecewise:

$$
\phi_{0}^{\dagger}(t)=\left\{\begin{array}{c}
\frac{t-m_{0}^{\dagger}}{K_{0} m_{0}^{\dagger}} f_{s}(t) \quad \text { if } t \in\left[m_{0}^{\dagger}, J m_{0}^{\dagger}\right] \\
\frac{J-1}{K_{0}} f_{s}(t) \quad \text { if } t \in\left[J m_{0}^{\dagger}, \infty\right)
\end{array}\right.
$$

where $m_{0}^{\dagger}$ is the unique value that satisfies $G^{\dagger}\left(m_{0}^{\dagger}\right)=1$. In addition, $m_{0}^{\dagger}<m_{0}^{*}$.

The authority intervenes with probability zero if $t<J m_{0}^{\dagger}$, and with probability

$$
\alpha(t)=1-\frac{J m_{0}^{\dagger}}{t} \quad \text { if } \quad t>J m_{0}^{\dagger}
$$

Payoffs. Type-0 trader's expected payoff is $u_{0}=m_{0}^{\dagger}(1-q)$. Authority's expected payoff is $1-q$.

Comparative Statics. The comparative statics with respect to $a, q$, and changes in the aggressiveness of the noise trade distribution are analogous to those in Proposition 4.1. An increase in $c$ increases $m_{0}^{\dagger}$ and $u_{0}$, and causes the type-0 trader to use a more aggressive trading strategy in the sense of monotone likelihood ratio. 
When $c>\widehat{\chi}$, sell orders are partitioned into two intervals, a safe zone of modest trades $\left(t \leq J m_{0}^{\dagger}\right)$ that never trigger an intervention and a risky zone of larger trades $\left(t>J m_{0}^{\dagger}\right)$ that trigger an intervention with positive probability. Over the safe zone the type- 0 trader mixes with a density similar to Proposition 4.1, and larger trades reveal more information, increasing the posterior belief $\chi(t)$. At the critical trade $t=J m_{0}^{\dagger}$, the authority is just indifferent about intervening, $\chi\left(J m_{0}^{\dagger}\right)=c$ (see Figure 2). At this point beliefs must stop increasing with order flow because higher beliefs would induce the authority to intervene with certainty. In order to truncate beliefs at this level, the informed trader pools with the noise trader over the risky zone of trades - inside the risky zone, the likelihood ratio $\phi_{0}(t) / f_{s}(t)$ is constant. Although her beliefs are constant over the risky zone, the probability that the authority intervenes increases with order flow to offset the investor's temptation to make larger trades.

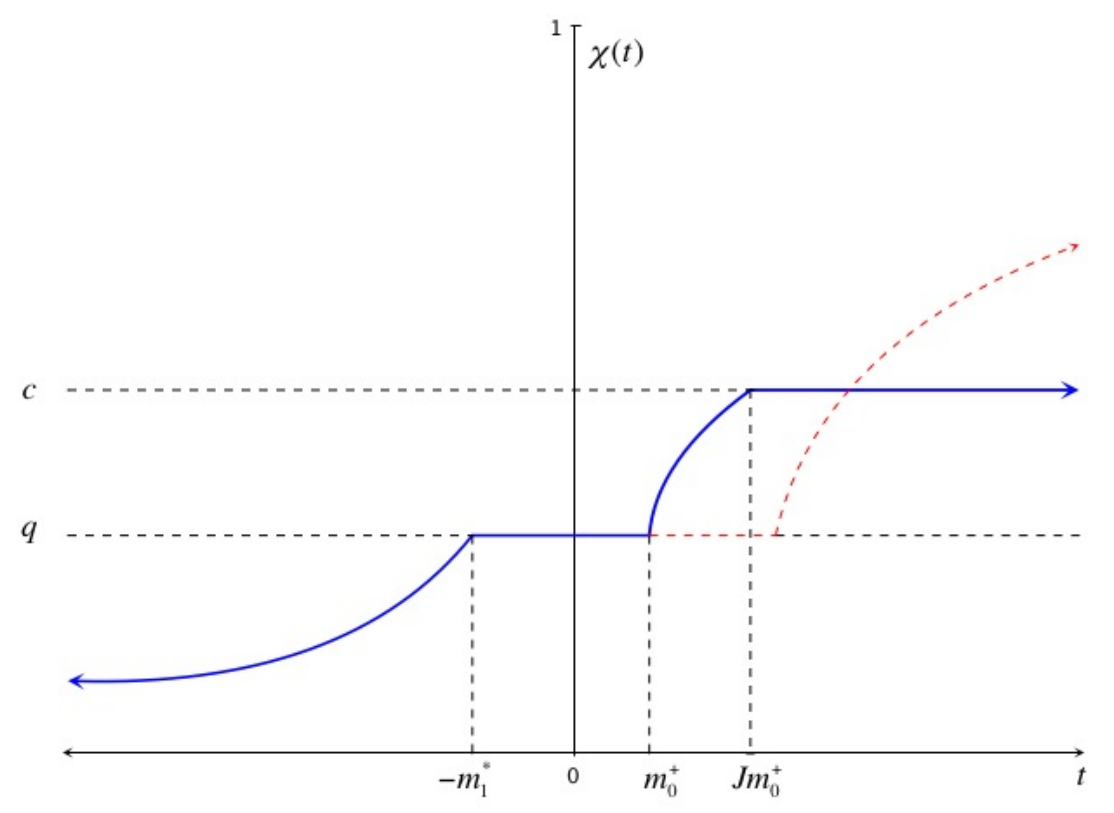

Figure 2: THE POSTERIOR BELIEF FOR THE EQUILIBRIUM WITH STOCHASTIC INTERVENTIONS

A degree of unpredictability is often inherent in both government intervention policies and corrective actions by firms. Examples include the differential treatments of Bear Stearns and Lehman Brothers, and the government's repeated refusal to delineate an explicit bailout policy for Fannie Mae and Freddie Mac (Frame and White 2005). Discussing firms' decisions to cancel mergers following negative market reaction, Luo (2005) presents several anecdotal examples of firms that cancelled their mergers, but he also presents one in which the firms did not cancel the deal. His empirical analysis also highlights the stochastic nature of the merger decision, consistent with the idea that the intervention (deal cancellation) is difficult to predict.

Unfortunately for the authority, when $c>\widehat{\chi}$, her expected equilibrium payoff is the same as 
if interventions were not possible. In the unique equilibrium, for every order, the authority either does not intervene, or mixes and is therefore indifferent between intervening and not. This is a direct consequence of the fundamental conflict of interest between the investor and the authority. Because the intervention completely erodes the value of the investor's private information, the investor avoids any order that triggers intervention for certain. At the same time, if the expected benefit of the intervention is positive, then the authority would like to undertake it for certain; thus no beneficial intervention could be triggered by a trader's order. In Section 7, we investigate a version of the model in which the authority can commit to an intervention policy that violates her sequential rationality condition (4). This commitment mitigates the conflict of interest, allowing the authority to benefit from the ability to intervene.

The type- 0 trader is worse off in the equilibrium of Proposition 5.2 than in the benchmark setting of Proposition 4.1: $m_{0}^{\dagger}(1-q)<m_{0}^{*}(1-q)$. In order to avoid triggering an intervention for certain with his order, the informed trader's mixing density is capped over the risky zone: he must put less mass on larger orders. Consequently his mixing distribution must be shifted towards smaller, less profitable trades. We show in the next proposition that the possibility of intervention induces him to trade less aggressively (in a formal sense) than he would if interventions were not possible. Because the equilibrium order flow acts as a public signal of the (pre-intervention) fundamental, less aggressive trade by the type-0 investor reduces the Blackwell-informativeness of this signal.

Proposition 5.3 (Equilibrium Properties). If $c \in(\widehat{\chi}, 1)$, then the following properties hold.

- (No benefit from intervention). The authority does not benefit from the ability to intervene: her payoff in the equilibrium with stochastic interventions is the same as her payoff in the no-intervention benchmark.

- (Less aggressive trade). The type-0 investor trades less aggressively in the equilibrium with stochastic interventions than in the no-intervention benchmark: his equilibrium mixed strategy in the absence of interventions dominates his equilibrium mixed strategy in the stochastic intervention equilibrium in the sense of monotone likelihood ratio.

- (Less information). Order flow is less Blackwell informative about the underlying state in the equilibrium with stochastic interventions than in the no-intervention benchmark.

- (Higher expected price). The expected asset price is higher in the equilibrium with stochastic interventions than in the no-intervention benchmark.

- (Non-monotonic price). In the stochastic intervention equilibrium the asset price is a nonmonotonic function of the order flow.

The last two points of this proposition describe properties of the equilibrium price. To understand why the expected asset price is higher, note that the equilibrium price (see equation (1)) is composed of two distinct terms: the first term $1-\chi(t)$ is the posterior belief that $\omega=1$ in 
the absence of an intervention, while the second term $\alpha(t) \chi(t)$ reflects the impact of a possible intervention. Because $1-\chi(t)$ is the posterior belief that $\omega=1$ conditional on order flow $t$, which is itself random (as it derives from a mixed strategy), the Law of Iterated Expectations implies that the expected value of $1-\chi(t)$ is equal to $1-q$. This is true whether or not interventions occur with positive probability. In the equilibrium with stochastic interventions, however, $\alpha(t)$ is positive for $t>J m_{0}^{\dagger}$, so the second term in the expected price, $E[\alpha(t) \chi(t)]$ is also positive. Interestingly, the increase in the asset price cannot be attributed to a less aggressive selling strategy on the part of the type-0 investor: changes in the trader's selling strategy have no effect on the expected fundamental, which is equal to $1-q$. Rather, the increase in price is due to the expectation of corrective intervention, $E[\alpha(t) \chi(t)]$, which is positive and is built into the price. ${ }^{18}$

With interventions, the asset price is a non-monotonic function of the order flow (review (1)). Over the safe zone, the price falls with larger sell orders as the market maker becomes more convinced that $\omega=0$-in this case $\chi(t)$ increases while $\alpha(t)=0$. Over the risky zone, however, the price rises as the market maker becomes ever more convinced that an intervention is forthcominghere $\chi(t)=c$ but $\alpha(t)$ increases. Overall, the possibility of intervention dampens the price impact of sell orders. ${ }^{19}$ This non-monotonicity also suggests that inferences about the state based on the price alone may be problematic - a high asset price is associated both with a small selloff (not requiring an intervention) and a large one (requiring an intervention with positive probability in equilibrium). This raises the question of what would transpire if the authority only observed the asset price and not order flow, a question we address in the following section.

\section{EXTENSIONS OF THE BASELINE MODEL}

In this section we analyze two variants of the baseline model studied above. First, we consider a setting in which the authority observes the asset price but does not observe the order flow. Second, we consider a setting in which the informed investor faces competition from a mass of small informed traders. While both of these extensions deliver intriguing results, the preceding positive and normative findings are largely robust to these considerations.

\section{INTERVENTION BASED ONLY ON PRICE}

Consider our baseline model with the modification that the authority observes only the price at which the trade occurs. In particular she does not observe order flow. In the next proposition, we

\footnotetext{
${ }^{18} \mathrm{~A}$ number of empirical papers document a connection between the expectation of corrective intervention and a high asset price. O'Hara and Shaw (1990) show that congressional testimony by the Comptroller of the Currency that some banks are "too big to fail" caused equity prices to increase at several large banks. Gandhi and Lustig (2010) also show that announcements in support of bailouts increase bank equity prices and present broad range of evidence. Frame and White (2005) survey several investigations estimating that debt issued by Fannie Mae and Freddie Mac trades at interest rates $0.35-0.40 \%$ below its risk rating, resulting in a higher asset price. According to these authors "financial markets treat [Fannie and Freddie's] obligations as if those obligations are backed by the federal government" despite the fact that the government is under no legal obligation to intervene in the event of trouble.

${ }^{19}$ This result connects to empirical research which establishes that institutional selling has a smaller price impact than institutional buying (Chan and Lakonishok 1995).
} 
show that no equilibrium exists in which the market maker uses a deterministic pricing function for all sell orders. Nevertheless, the equilibrium price perfectly reveals all information about the state that the market maker gleans from the trader's order. Furthermore, the trader's mixed strategy, the probability of intervention conditional on order $t$, and the expected price conditional on order $t$ are all identical to the main model.

For purposes of this subsection, let the probability of intervention following price $p$ be denoted $\beta(p)$, and let $\chi_{A}(p)$ be the authority's belief that the state is bad conditional on observing price $p$. As in the preceding sections, we assume that the market maker generates zero profit, executing the trader's order at a price that is equal to the asset's expected value. That is, if order $t$ is executed at price $p$ in equilibrium, then

$$
p=(1-\beta(p))(1-\chi(t))+\beta(p) .
$$

Note that the market maker observes the trader's order $t$, and his equilibrium belief about the state conditional on $t$ is $\chi(t)$, as in equation (6).

Proposition 6.1 (Intervention based only on price) If $c \in(\widehat{\chi}, 1)$ and the authority observes the asset price but not the order flow, then a unique equilibrium exists, which is expected payoff equivalent for all parties to the one given in Proposition 5.2.

Trader Strategy. The type-0 trader uses the same mixing density $\phi_{0}^{\dagger}(t)$ as in Proposition 5.2 and the type-1 trader uses the same mixing density $\phi_{1}^{*}(t)$ as in Proposition 4.1.

Authority Strategy. In equilibrium, if $p \in[1-c, 1)$, then the authority does not intervene $\beta(p)=0$, and if $p=1$, then the authority intervenes for certain, $\beta(p)=1$.

Market Maker Behavior. For all buy orders $t \in(-\infty, 0]$ the market maker sets the price in the same way as in Proposition 4.1 and for all sell orders $t \in\left[0, J m_{0}^{\dagger}\right)$ he sets the price in the same way as in Proposition 5.2, namely $p(t)=1-\chi(t) \in(1,1-c)$. For each sell order $t \in\left[\mathrm{Jm}_{0}^{\dagger}, \infty\right)$, the market maker's price is the realization of random variable $P_{t}$, where

$$
P_{t}=\left\{\begin{array}{ccc}
1 & \text { with probability } & 1-\frac{J m_{0}^{\dagger}}{t} \\
1-c & \text { with probability } & \frac{J m_{0}^{\dagger}}{t} .
\end{array}\right.
$$

All prices $p \in[1-c, 1]$ are on the equilibrium path.

Information Transmission. For every price on the equilibrium path, the authority's updated belief about the state conditional on the price is identical to the market maker's belief about the state conditional on the trader's order, $\chi_{A}(p)=\chi(t)$.

While many features of the equilibrium are identical to the case in which the order flow is observable, the equilibrium also exhibits a crucial difference: the market maker must execute sell orders in the risky zone at a random price. In particular, for $t>J m_{0}^{\dagger}$ the market maker 
randomizes between the two extreme prices $p=1-c$ and $p=1$. In either case the authority infers $\chi_{A}(1)=\chi_{A}(1-c)=c$, leaving her indifferent. She responds by intervening with probability 1 when observing $p=1$ and with probability 0 when observing $p=1-c$. That is, a price of 1 communicates that the market anticipates an intervention, while a price of $1-c$ communicates that no intervention is anticipated. The probability that the market maker selects $p=1$ for order $t$ in the risky zone is identical to the probability that the authority intervenes following order $t$ in the equilibrium with observable order flow. In essence, the market maker adopts the equilibrium strategy played by the authority when the order flow is observable, while the authority passively implements the intervention policy implied by the price. This result reflects a tension that is generated by the unobservability of the trader's order: first, the price must incorporate both the market maker's information about the asset and the probability of intervention. Second, the asset price conveys information about the fundamental to the authority, informing her of the appropriate intervention policy. This dual role of prices cannot be fulfilled if the market maker always sets the price deterministically.

To see this intuitively, suppose first that the market maker uses a deterministic pricing rule that is monotonic inside the support of type-0's mixed strategy. In this case the authority would invert the pricing function to determine the order size and the threshold for intervening. But then the equilibrium would revert to Proposition 5.2, which generates a non-monotonic pricing function. A monotonic price function accurately conveys information to the authority but it does not correctly price the asset because it cannot incorporate the intervention's expected impact. If instead the market maker's pricing function is non-monotonic inside the support of type-0's mixed strategy, then multiple orders inside the support generate the same price, and-because the order is not observed by the authority - the same probability of intervention. But then the trader strictly prefers the larger order, violating the indifference condition inside the support of his mixed strategy. A non-monotonic pricing function can incorporate the probability of intervention to accurately reflect the asset's value, but this interferes with its ability to guide the authority's intervention.

The equilibrium price is depicted in Figure 3. The brown curve represents the pricing function with observable order flow. With unobservable order flow, the price is identical inside the safe zone, $t<J m_{0}^{\dagger}$. For $t>J m_{0}^{\dagger}$, the market maker randomizes between $p=1$ and $p=1-c$, which are depicted in orange. The market maker's randomization is done in such a way that the expected price at which order $t$ is cleared in the unobservable case is identical to the price at which it is cleared in the observable case. This probability is the height of the purple segment divided by $c$. 


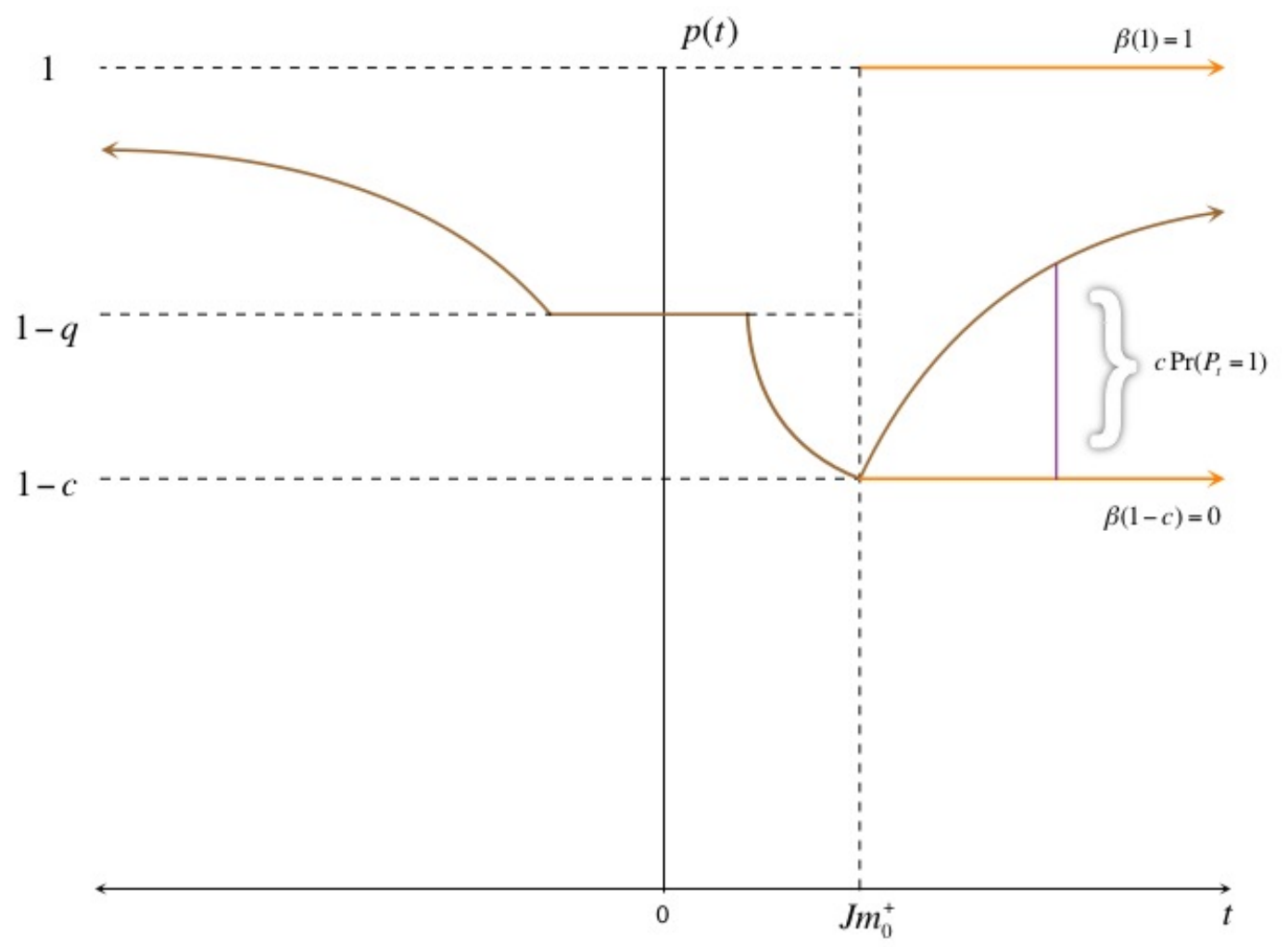

Figure 3: Price function in the observable And unobservable CASES.

\section{MULTIPLE INFORMED TRADERS}

Throughout the analysis we have assumed that the informed trader is the only investor with information about the state. However, if multiple informed investors are present, then each one plays a smaller role in the market, which has the potential to affect the results of our baseline model.

It is possible to introduce multiple traders into our model in several ways. In this subsection we analyze the simplest possible extension of the basic model. Specifically, we suppose that there are two groups of informed traders, a single large investor who is unconstrained in his trading opportunities and a competitive fringe of small investors who face liquidity constraints and can trade only a single massless share of the asset. ${ }^{20}$ We assume that the competitive fringe of informed traders is present if and only if the large informed investor is present.

We denote the commonly known measure of the competitive fringe by $y \in(0, \infty)$ and the quantity traded by the large informed investor by $x \in \Re$. Note that for a trader in the fringe, investing is a weakly dominant strategy. ${ }^{21}$ Hence, the competitive fringe invests with probability 1 ,

\footnotetext{
${ }^{20}$ Studying a version of our model with multiple large informed investors would be interesting, but dealing with the significant technical strategic details would divert the focus of this investigation. We therefore, leave such a version of the model for future work.

${ }^{21}$ Let $t$ represent a realization of the total order flow. If the state is one and he invests, then the fringe investor
} 
generating order $y$ in state 0 and $-y$ in state 1 . Therefore, the total informed order flow is $t=x+y$ in state zero and $t=x-y$ in state one. The market maker and authority observe only the total order flow. Let $\phi_{i}(x, y)$ denote the type- $i$ informed trader's mixing density when the size of the competitive fringe is $y{ }^{22}$ Conditional on total sell order flow $t>0$, the updated belief that the state is zero is

$$
\chi(t)=\frac{q\left(a \phi_{0}(t-y, y)+(1-a) s f_{s}(t)\right)}{q a \phi_{0}(t-y, y)+(1-a) s f_{s}(t)} \quad \text { if } t>0
$$

and conditional on total buy order flow $t<0$,

$$
\chi(t)=\frac{q(1-a)(1-s) f_{b}(t)}{q a \phi_{1}(t+y, y)+(1-a)(1-s) f_{b}(t)} \quad \text { if } t<0 .
$$

In essence, the market maker compares the likelihood that the trade was submitted by the noise trader to the likelihood that the order is a batch of the large investor and the competitive fringe. The price is set as in equation (1), and the large informed investor's payoff of submitting order $x \in \Re$ is

$$
u_{0}(x)=x(1-\chi(x+y))(1-\alpha(x+y)) \quad u_{1}(x)=-x \chi(x-y)(1-\alpha(x-y)) .
$$

Following an analogous argument to Proposition 4.1, we derive the following result.

Proposition 6.2 (Competitive fringe, no interventions). The unique equilibrium with a competitive fringe of measure $y$ when interventions are not possible is characterized as follows. Let

$$
g_{s}^{*}(m, y) \equiv \int_{m+y}^{\infty} \frac{t-m-y}{K_{0} m} f_{s}(t) \mathrm{d} t \quad \text { and } \quad g_{b}^{*}(m, y) \equiv \int_{m+y}^{\infty} \frac{t-m-y}{K_{1} m} f_{b}(-t) \mathrm{d} t .
$$

Strategies. Type-0 plays a mixed strategy $\phi_{0}^{*}(x, y)$ on support $\left[m_{0}^{*}(y), \infty\right)$, and type-1 plays a mixed strategy $\phi_{1}^{*}(t, y)$ on support $\left(-\infty,-m_{1}^{*}(y)\right]$, where

$$
\phi_{0}(x, y)=\frac{x-m_{0}^{*}(y)}{K_{0} m_{0}^{*}(y)} f_{s}(x+y) \text { and } \phi_{1}(x, y)=\frac{|x|-m_{1}^{*}(y)}{K_{1} m_{1}^{*}(y)} f_{b}(x-y)
$$

and $\left(m_{0}^{*}(y), m_{1}^{*}(y)\right)$ are the unique values that satisfy $g_{s}^{*}\left(m_{0}^{*}(y), y\right)=1$ and $g_{b}^{*}\left(m_{1}^{*}(y), y\right)=1$.

Payoffs. The type-i large informed trader's payoff is $u_{i}=m_{i}^{*}(y)(i q+(1-i)(1-q))$.

Comparative Statics. An increase in y (i) reduces $m_{i}^{*}(y)$ and the expected profit of the large informed investor, (ii) increases the minimum total informed order size $m_{i}^{*}(y)+y$, (iii) reduces the type-i large informed investor's order size in the sense of monotone likelihood ratio if $f_{i}(\cdot)$ is log-concave, (iv) increases the total order size in the sense of monotone likelihood ratio.

expects payoff $1-p(t)$, and if the state is zero and he invests, then the fringe investor expects $p(t)-\alpha(t)$. Equation (1) implies that $p(t) \in[\alpha(t), 1]$, and thus, investing is weakly dominant for a trader in the fringe.

${ }^{22}$ Mass points in the informed trader's mixing distribution can be ruled out as in Section 5 . 
In equilibrium the large informed investor's mixing density is a transformation of the noise trader's mixing density incremented by $y$, which accounts for the competitive fringe's contribution to the total order flow. Naturally, the competitive fringe undermines the large informed trader's opportunity to profit from his private information, reducing his equilibrium payoff, which is driven to zero as the competitive fringe grows, $y \rightarrow \infty$. If the noise trader's sell order flow is log-concave, then the large informed trader also trades less aggressively as the size of the fringe increases, but the reduction in the large trader's aggressiveness is offset by the competitive fringe, resulting in a total equilibrium order flow that is larger in the sense of monotone likelihood ratio and more informative in the sense of Lehmann (1988) and Blackwell.

If the cost of intervention is too low, then the authority intervenes with probability one following any sell order flow that could originate from the large informed trader, i.e. exceeds $y$. As in the preceding section, we show that this happens in equilibrium whenever the cost of intervention is smaller than the Bayesian update that $\omega=0$, given a sell order larger than $y$. That is, in equilibrium any sell order larger than $y$ triggers an intervention with probability one whenever

$$
c<\widehat{\chi}(y) \equiv \frac{a q+q s(1-a)\left(1-F_{s}(y)\right)}{a q+s(1-a)\left(1-F_{s}(y)\right)}=\frac{K_{0}+q\left(1-F_{s}(y)\right)}{K_{0}+\left(1-F_{s}(y)\right)} .
$$

As above, we focus on the more interesting case in which an intervention is not triggered with probability one whenever the traders are informed, $c>\widehat{\chi}(y)$. Note that $\widehat{\chi}(y)$ is increasing in $y$, and approaches one as $y \rightarrow \infty$. Therefore, (15) can also be interpreted as a restriction on the size of the competitive fringe. If it is too large, then the competitive fringe's trade, on its own, is sufficiently informative to generate an intervention. In this case, the value of the large informed trader's information is driven to zero; i.e., there are no profitable trades he can make.

Proposition 6.3 (Competitive fringe, stochastic interventions). When $c \in(\widehat{\chi}(y), 1)$, interventions are possible, and a competitive fringe of size y exists, the game has a unique equilibrium, characterized as follows. Let

$$
g^{\dagger}(m, y) \equiv \int_{m+y}^{J m+y} \frac{t-m-y}{K_{0} m} f_{s}(t) \mathrm{d} t+\frac{J-1}{K_{0}}\left(1-F_{s}(J m+y)\right)
$$

Strategies. The type-0 trader places a sell order distributed according to continuous probability density function $\phi_{0}(x, y)$ over support $\left[m_{0}^{\dagger}(y), \infty\right)$ defined piecewise:

$$
\phi_{0}(x, y)=\left\{\begin{array}{cl}
\frac{x-m_{0}^{\dagger}(y)}{K_{0} m_{0}^{\dagger}(y)} f_{s}(x+y) & \text { if } x \in\left[m_{0}^{\dagger}(y), J m_{0}^{\dagger}(y)\right] \\
\frac{J-1}{K_{0}} f_{s}(x+y) & \text { if } x \in\left[J_{0}^{\dagger}(y), \infty\right)
\end{array}\right.
$$

where $m_{0}^{\dagger}(y)$ is the unique value that satisfies $g^{\dagger}\left(m_{0}^{\dagger}(y), y\right)=1$. In addition, $m_{0}^{\dagger}(y)<m_{0}^{*}(y)$. The 
authority intervenes with probability zero if $t \leq J m_{0}^{\dagger}(y)+y$, and with probability

$$
\alpha(t, y)=1-\frac{J m_{0}^{\dagger}(y)}{t-y} \quad \text { if } \quad t>J m_{0}^{\dagger}(y)+y
$$

Payoffs. Type-0 trader's expected payoff is $m_{0}^{\dagger}(y)(1-q)$. Authority's expected payoff is $1-q$.

Comparative statics. An increase in y that does not violate (15): (i) reduces $m_{0}^{\dagger}(y)$ and the expected profit of the type-0 large investor, (ii) increases $m_{0}^{\dagger}(y)+y$ and decreases $J_{0}^{\dagger}(y)+y$, shrinking the safe zone and expanding the risky zone, (iii) reduces the type-0 large informed investor's order flow in the sense of monotone likelihood ratio if $f_{s}(\cdot)$ is log-concave, (iv) weakly increases the probability of intervention for each order flow and the ex ante probability of intervention.

The equilibrium responses to the introduction of a competitive fringe of informed traders is intuitive whether or not interventions are possible. Specifically, the competitive fringe undermines the large informed trader's profit and dissipates it completely if the fringe is sufficiently massive. In other words, competition among informed investors naturally erodes profitable trading opportunities. Nevertheless, Propositions 6.2 and 6.3 indicate that the positive and normative effects generated by the possibility of intervention characterized in Proposition 5.3 are robust to a simple form of competition between informed traders, provided that the degree of competition is not too high.

\section{NORMATIVE REMEDIES}

As noted in Proposition 5.2, when $c \in(\widehat{\chi}, 1)$, the authority intervenes stochastically, but does not benefit relative to the no-intervention benchmark. The reason for this is her lack of commitment power: it is sequentially rational for the authority to intervene if $\chi(t)>c$. The type-0 trader therefore chokes off the information content of order flow for $t>J m_{0}^{\dagger}$ to avoid triggering a certain intervention, which would deprive him of all rent.

In this section we first explore a benchmark setting in which ex ante commitment to an intervention policy $\alpha(t)$ is possible. In particular, we derive the ex ante optimal intervention plan for the authority holding the type-0 investor's expected payoff at its equilibrium level, $u_{0}=m_{0}^{\dagger}(1-q)$. This restriction ensures that the expected payoffs of all market participants are unchanged from their equilibrium values so that the policy we derive represents a Pareto improvement over the equilibrium. $^{23}$ Although we do not regard full commitment to an optimal random intervention policy as especially realistic, it is helpful to understand how the authority's optimal plan differs from her no-commitment equilibrium strategy. Further, this comparison paves the way for more plausible institutional remedies that we consider subsequently.

\footnotetext{
${ }^{23}$ Recall that the market maker's payoff is zero, and informed trader rents come at the expense of noise traders. Other Pareto optimal policies exist (indexed by the informed trader's payoff) but these cannot be Pareto ranked.
} 


\section{PARETO IMPROVING INTERVENTIONS}

To formulate the authority's optimal policy with commitment as a constrained programming problem, we adopt the standard approach of the principal-agent literature, allowing the authority to select both her own strategy and the type- 0 trader's, imposing the equilibrium conditions for the trader's strategy as incentive compatibility constraints. We therefore imagine that the authority chooses $\alpha(t)$ and $\phi_{0}(t)$ in order to maximize her ex ante expected payoff

$v=(1-q)(a+(1-a)(1-s))+\int_{0}^{\infty}\left(a q \phi_{0}(t)+(1-a) s f_{s}(t)\right)(\alpha(t)(1-c)+(1-\alpha(t))(1-\chi(t))) \mathrm{d} t$.

The first term in this expression is the contribution to the authority's expected payoff from a buy order (after which it is never optimal to intervene). The first term of the integrand is the density of sell orders and the second term is the authority's expected payoff given the chosen intervention probability at order flow $t$. This maximization is subject to feasibility conditions that ensure that the intervention probability and mixing density are valid, incentive constraints, that ensure that the trader is willing to comply with the authority's recommended mixing density, and a constraint that ensures that the informed trader's payoff is unaffected by the authority's commitment power (see the proof of Proposition 7.1 for details). The following proposition describes the solution in the most intuitive case, $J>2$. Propositions B.8 and B.9 (in the appendix) provide a characterization for all values of $J$.

Proposition 7.1 (Pareto Improving Interventions). Suppose $J>2$ and $c \in(\widehat{\chi}, 1)$. For each $\left(J, K_{0}\right)$, there exists a $\lambda \in(0,1)$ such that the solution of the authority's maximization problem is characterized as follows. The type-0 trader places a sell order distributed according to continuous probability density function $\widehat{\phi}_{0}(t)$ over support $\left[m_{0}^{\dagger}, \infty\right)$ defined piecewise:

$$
\widehat{\phi}_{0}(t)=\left\{\begin{array}{cl}
\frac{t-m_{0}^{\dagger}}{K_{0} m_{0}^{\dagger}} f_{s}(t) & \text { if } t \in\left[m_{0}^{\dagger}, \frac{J m_{0}^{\dagger}}{1+\lambda}\right] \\
\left(\frac{J-1}{K_{0}}+\frac{(1-\lambda) t-J m_{0}^{\dagger}}{2 K_{0} m_{0}^{\dagger}}\right) f_{s}(t) & \text { if } t \in\left[\frac{J m_{0}^{\dagger}}{1+\lambda}, \infty\right) .
\end{array}\right.
$$

The authority intervenes with probability $\widehat{\alpha}(t)=\frac{1}{2}\left(1+\lambda-\frac{J m_{0}^{\dagger}}{t}\right)$ if $t>\frac{J m_{0}^{\dagger}}{1+\lambda}$ and 0 otherwise.

When designing the ex ante optimal policy, the authority faces a delicate tradeoff. In order to induce the type-0 trader to reveal information, she must commit not to use the information too aggressively. On the other hand, acquiring information is pointless if she cannot act on it by making a beneficial intervention. To highlight this tension, note that (17) simplifies to: ${ }^{24}$

$$
v=(1-q)+a q(1-c) \int_{0}^{\infty} \alpha(t)\left(\phi_{0}(t)-\frac{J-1}{K_{0}} f_{s}(t)\right) \mathrm{d} t
$$

\footnotetext{
${ }^{24}$ See the proof of proposition 7.1 for the derivation.
} 
Two things are evident from this formulation of the authority's objective. First, it is clear why she does not benefit in an equilibrium without commitment - namely, $\alpha(t)=0$ for $t<J m_{0}^{\dagger}$ and $\phi_{0}(t)=\frac{J-1}{K_{0}} f_{s}(t)$ for $t \geq J m_{0}^{\dagger}$; therefore $v=1-q$ in this case. Second, an intervention at $t$ is beneficial if and only if $\phi_{0}(t)>\frac{J-1}{K_{0}} f_{s}(t)$. The incentive constraints of the trader, however, imply that a high value of $\phi_{0}(t)$ necessitates a low value of $\alpha(t)$ and vice versa: acquiring valuable information requires a commitment to intervene with relatively low probability.

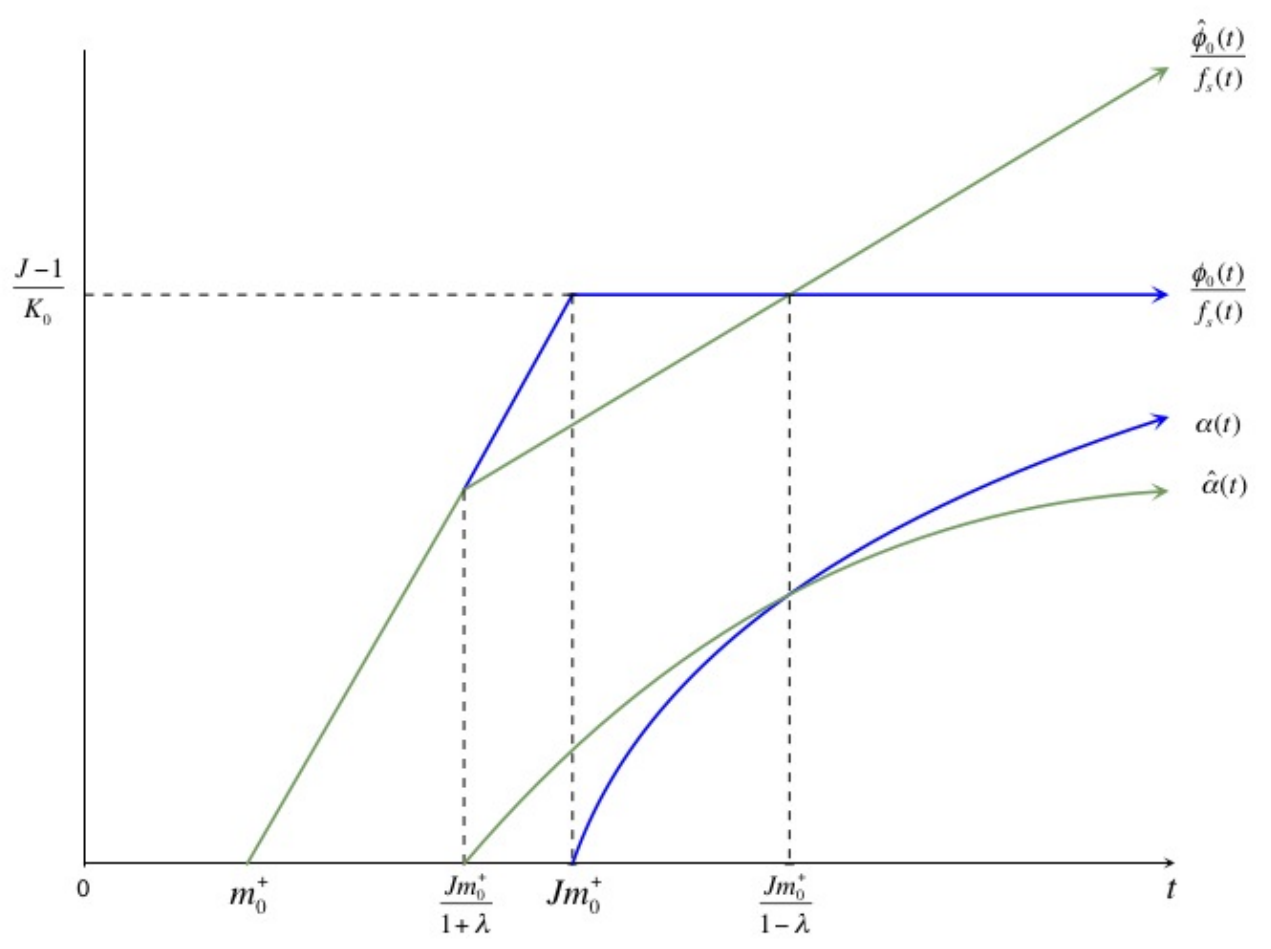

Figure 4: The Pareto Optimal Intervention Policy

As illustrated in Figure 4, compared with the authority's strategy in the stochastic interventions equilibrium of Proposition 5.2, her ex ante optimal intervention policy (viewed as a function of the order flow) has a smaller intercept and is less steep. The authority therefore optimally begins intervening sooner (i.e. for smaller sell orders), but uses an intervention rule that is flatter and therefore less sensitive to increases in the trader's order. The two intervention policies intersect at order flow $t=J m_{0}^{\dagger} /(1-\lambda)$; for larger order flows the optimal policy lies below the equilibrium one (and is weakly above for smaller orders).

Facing the authority's ex ante optimal intervention policy, the type-0 trader mixes according to a density that is a counterclockwise rotation of the no-commitment equilibrium strategy. In particular, this density is the same as his no-commitment equilibrium strategy over the commitment safe zone, $\left[m_{0}^{\dagger}, J m_{0}^{\dagger} /(1+\lambda)\right]$. However, the commitment safe zone is smaller than the safe zone without commitment, ending at order flow $t=J m_{0}^{\dagger} /(1+\lambda)$ instead of $t=J m_{0}^{\dagger}$. At $t=J m_{0}^{\dagger} /(1+\lambda)$ 
the type-0 trader's optimal density becomes flatter so that the authority's belief increases less rapidly as the order flow increases. The optimal density continues increasing until it crosses the density $\phi_{0}(t)$ at $J m_{0}^{\dagger} /(1-\lambda)$. Thus, for order flow $t \in\left(J m_{0}^{\dagger} /(1+\lambda), J m_{0}^{\dagger} /(1-\lambda)\right)$ beliefs satisfy $\chi(t)<c$ and interventions - which occur with positive probability under the optimal policy actually harm the authority in expectation. On the other hand, for order flow $t \in\left(J m_{0}^{\dagger} /(1-\lambda), 1\right]$, beliefs satisfy $\chi(t)>c$ and interventions strictly benefit the authority in expectation.

The ex ante Pareto optimal policy therefore requires both kinds of commitment from the authority: for an intermediate range of order flow she must intervene with positive probability when she would prefer not to intervene at all, and for high order flow she must refrain from intervening with certainty, although she would benefit from doing so. In essence, the optimal intervention policy is a clockwise rotation of the equilibrium policy that induces a counter-clockwise rotation in the trader's mixing density over the region $\left[J m_{0}^{\dagger} /(1+\lambda), 1\right]$. Thus, the optimally induced mixing density for the trader conveys more information on large orders, increasing the authority's payoff from an intervention. For this to be incentive compatible, the authority must intervene less often on large orders and slightly more often for an interval of moderate orders. Although the characterization presented in Proposition 7.1 assumes $J>2$, these rotation properties of the optimal commitment policy also hold for $J<2$.

Proposition 7.2 (Commitment and Rotation). The intervention policy in the equilibrium with commitment, $\widehat{\alpha}(t)$ can be obtained from the intervention policy in the equilibrium without commitment, $\alpha(t)$, via a clockwise rotation. The type-0 trader's mixing density in the equilibrium with commitment, $\widehat{\phi}_{0}(t)$, can be obtained from the intervention policy in the equilibrium without commitment, $\phi_{0}(t)$, via a counterclockwise rotation. That is, there exists $\widehat{t}>m_{0}^{\dagger}$ such that

$$
\begin{aligned}
& t>\widehat{t} \Rightarrow \widehat{\alpha}(t)<\alpha(t), \text { and } \widehat{\phi}_{0}(t)>\phi_{0}(t), \\
& t<\widehat{t} \Rightarrow \widehat{\alpha}(t)>\alpha(t), \text { and } \widehat{\phi}_{0}(t) \leq \phi_{0}(t) .
\end{aligned}
$$

Of course, committing to an intervention policy that randomizes with precisely the correct probabilities at each possible value of the order flow is implausible because it requires verification by the type- 0 trader or some impartial third party. In the absence of such verification, the authority could simply implement her ex post preferred policy and claim that she randomized according to the ex ante optimal one. Even if the authority cannot commit to the precise ex ante optimal intervention policy, she may be able to verifiably commit to an institution that - while suboptimal - still raises her expected payoff. We investigate two possibilities below.

\section{IMPERFECT INTERVENTIONS}

Here we consider an alternative environment in which the institution or technology that executes interventions is imperfect. The institution caps the intervention probability from above at some commonly known level $\bar{\alpha}<1$. For example, it could be that an attempted intervention is blocked with probability $1-\bar{\alpha}$, so that the probability of a successful intervention given one is attempted 
with probability $\beta(t)$ is $\alpha(t)=\bar{\alpha} \beta(t)$. Alternatively, the authority could employ an intervention technology that does not guarantee that the state is one for certain, but effects a transition to state one with some probability $\bar{\alpha}<1$. Because the cap is a feature of the institution or technology, its existence is likely to be common knowledge among all parties.

As noted in Proposition 7.1, the Pareto optimal policy is flatter than the equilibrium policy. A cap on the intervention policy approximates this by forcing the authority to use a completely flat intervention policy for large orders (where the cap is binding). With the cap imposed, sequential rationality for the authority requires:

$$
\alpha(t)=\left\{\begin{array}{ccc}
0 & \text { if } & \chi(t)<c \\
{[0, \bar{\alpha}]} & \text { if } & \chi(t)=c \\
\bar{\alpha} & \text { if } & \chi(t)>c
\end{array}\right.
$$

This condition suggests why the cap might be desirable for the authority. Without the cap, whenever the authority believes that intervention is strictly beneficial, she intervenes with probability one, depriving the trader of all rent (see equation (2)). The trader therefore mixes in a way that chokes off information and avoids a certain intervention. With the cap, the authority cannot intervene with probability one and leave the trader with zero payoff. Therefore, the trader may be willing to place more weight on large orders, even if this leads the authority to believe that an intervention is strictly beneficial. This intuition is formalized in the following proposition.

Proposition 7.3 (Imperfect Interventions). If $c \in(\widehat{\chi}, 1)$ and $\bar{\alpha}<1$, then the game has a unique equilibrium that is characterized below. Let

$$
\bar{G}(m) \equiv \int_{m}^{J m} \frac{t-m}{K_{0} m} f_{s}(t) \mathrm{d} t+\frac{J-1}{K_{0}}\left(F_{s}\left(\frac{J m}{1-\bar{\alpha}}\right)-F_{s}(J m)\right)+\int_{\frac{J m}{1-\bar{\alpha}}}^{\infty} \frac{t(1-\bar{\alpha})-m}{K_{0} m} f_{s}(t) \mathrm{d} t .
$$

Strategies. The type-0 trader places a sell order distributed according to continuous probability density function $\phi_{0}(t)$ over support $\left[\bar{m}_{0}, \infty\right)$ defined piecewise:

$$
\phi_{0}(t)=\left\{\begin{array}{cll}
\frac{t-\bar{m}_{0}}{K_{0} \bar{m}_{0}} f_{s}(t) & \text { if } t \in\left[\bar{m}_{0}, J \bar{m}_{0}\right] \\
\frac{J-1}{K_{0}} f_{s}(t) & \text { if } t \in\left[J \bar{m}_{0}, \frac{J \bar{m}_{0}}{1-\bar{\alpha}}\right] \\
\frac{(1-\bar{\alpha}) t-\bar{m}_{0}}{K_{0} \bar{m}_{0}} f_{s}(t) & \text { if } t \in\left[\frac{J \bar{m}_{0}}{1-\bar{\alpha}}, \infty\right)
\end{array}\right.
$$

where $\bar{m}_{0}$ is the unique value that satisfies $\bar{G}\left(\bar{m}_{0}\right)=1$. In addition, $\bar{m}_{0} \in\left[m_{0}^{\dagger}, m_{0}^{*}\right]$. The authority intervenes with probability zero if $t<J \bar{m}_{0}$, and with probability

$$
\alpha(t)=\left\{\begin{array}{cl}
1-\frac{J \bar{m}_{0}}{t} & \text { if } \quad t \in\left[J \bar{m}_{0}, \frac{J \bar{m}_{0}}{1-\bar{\alpha}}\right] \\
\bar{\alpha} & \text { if } \quad t \in\left[\frac{J \bar{m}_{0}}{1-\bar{\alpha}}, \infty\right)
\end{array}\right.
$$

Payoffs. The type-0 trader's expected payoff is $u_{0}=\bar{m}_{0}(1-q)$. The authority's expected payoff is strictly greater with a binding cap $(\bar{\alpha}<1)$ than without a binding cap $(\bar{\alpha}=1)$. 
When interventions are imperfect, the risky zone of trades is split into two parts. For $t \in$ $\left[J \bar{m}_{0}, J \bar{m}_{0} /(1-\bar{\alpha})\right]$, the cap on the intervention probability does not bind, and equilibrium behavior for both players is similar to that given in Proposition 5.2. The informed investor chokes off information, mixing proportionally to the noise density, and the authority intervenes with increasing probability. For the higher range of orders, $t>J \bar{m}_{0} /(1-\bar{\alpha})$, the cap on intervention probability binds. Over this range, the type-0 investor mixes using an increasing density, thereby releasing more information than in the case with no cap; indeed over this range $\chi(t)>c$, and because interventions take place with positive probability over this range, the authority expects to benefit (see Figure 5).

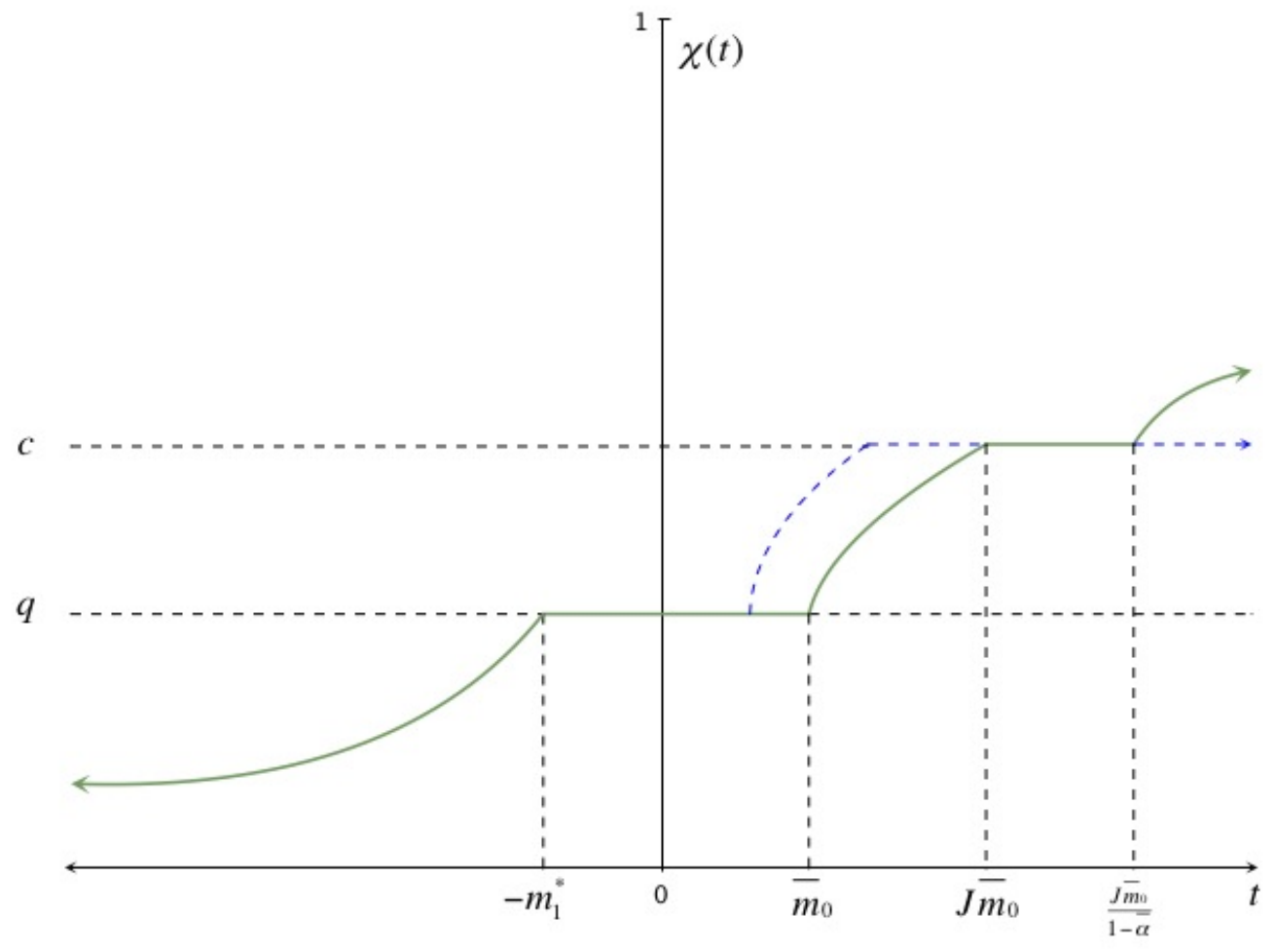

Figure 5: THE POSTERIOR BELIEF IN THE EQUILIBRIUM WITH A BINDING INTERVENTION CAP.

The imperfect technology commits the authority not to intervene with certainty, and this induces the investor to reveal more information over the interval of extreme trades where the cap binds. Because large orders simultaneously convey more information and sometimes trigger interventions, the authority benefits relative to the case of stochastic interventions with no cap. In fact, both the authority and the type-0 trader prefer that the authority uses any (sufficiently) imperfect intervention technology.

As $\bar{\alpha}$ ranges from 0 to 1 , the equilibrium moves continuously from the no-intervention benchmark of Proposition 4.1 to the stochastic intervention setting of Proposition 5.2. Since the type-0 trader 
prefers the former environment to the latter one, it is not surprising that his welfare increases as the cap decreases. Recall, however, that the authority's expected payoff is $1-q$ in both the no-intervention and stochastic-intervention settings. Nevertheless, her expected equilibrium payoff is strictly higher for any intermediate case. The reason is that the cap $\bar{\alpha}$ influences her payoff in two ways. With a tighter cap, the investor reveals more information through his trades, but the authority is less able to use this information to execute a beneficial intervention. At one extreme $\bar{\alpha}=0$, the investor reveals the most information, but the authority's hands are tied. At the other extreme $\bar{\alpha}=1$, the authority is unconstrained, but the investor reveals no valuable information. For all intermediate values of the cap, the investor reveals some valuable information which the authority can sometimes exploit.

Unlike the Pareto optimal policy of Proposition 7.1 which improves the authority's payoff while keeping the market participants' payoffs at their equilibrium levels, imperfect interventions benefit the informed trader as well as the authority. This benefit to the informed trader comes at the expense of the noise trader, however, so that the capped equilibrium does not Pareto dominate the stochastic intervention equilibrium. Imperfections in the decision process or intervention technology benefit the authority and increase utilitarian social welfare but also effect a "transfer" of utility from the noise trader to the informed trader.

\section{DELEGATING AUTHORITY}

Imagine that the authority delegates the decision to deploy the intervention to an agent. The agent shares the authority's preferences for the fundamental (receiving payoff one if the terminal fundamental is $\omega=1$ ) and also internalizes the full cost of the intervention, $c$, (which we assume is borne by the authority). Unlike the authority, whose preferences depend only on the state and on the intervention cost, the agent's preferences also place positive weight, $b(1-c) / q$, on the noise trader's realized profit. ${ }^{25}$ The term $(1-c) / q$ is a convenient normalization, while $b>0$ captures the degree of the agent's bias. The agent has exactly the same information as the authority: she does not know the trader's type at the time that she decides whether to intervene, though she draws inferences about the trader's type from the observed order. We characterize the resulting equilibrium, showing that the authority benefits from this type of delegation provided that the agent's bias is not too large.

To begin the analysis, define the agent's posterior belief that the investor is a noise trader conditional on observing sell order $t$ by

$$
\eta(t) \equiv \frac{(1-a) s f_{s}(t)}{(1-a) s f_{s}(t)+a q \phi_{0}(t)}=\frac{f_{s}(t)}{K_{0} \phi_{0}(t)+f_{s}(t)} .
$$

To write the agent's expected payoff, observe that if the agent intervenes with probability $\alpha$ following an executed sale of size $t$ transacted at price $p(t)$, then the expected value of the noise trader's

\footnotetext{
${ }^{25}$ Although we model the agent as weighting only the noise trader's profit, similar results hold if the agent weights the informed trader's profit, or weights both. Protection of noise traders - who trade in response to liquidity shocksis a plausible goal both for government regulators and firm managers.
} 
terminal profit is $\pi_{N}(t) \equiv t(p(t)-(1-\alpha)(1-q)-\alpha) \cdot{ }^{26}$ Indeed, if the noise trader submits a sell order, he collects $p(t)$ on each share, but must pay back one if the agent intervenes, or if the agent does not intervene but the state is good. Therefore, intervening (after the sell order has been executed) reduces the noise trader's profit, because it forces him to pay back 1 per share with probability 1 , instead of probability $1-q$. Thus, the agent's expected payoff from intervening with probability $\alpha$ after observing a sale of size $t$ executed at price $p(t)$ is

$$
\{1-\chi(t)+\alpha(\chi(t)-c)\}+\left(b \frac{1-c}{q}\right) \eta(t) \pi_{N}(t) .
$$

The bracketed term represents the authority's payoff and the final term represents the agent's weight on the informed trader's ex post payoff. Belief function $\eta(t)$ represents the agent's uncertainty about whether the investor is the noise trader conditional on the observed order flow.

By maximizing the agent's expected payoff with respect to $\alpha$, we find her sequentially rational intervention strategy:

$$
\text { for sell order } t, \quad \widetilde{\alpha}(t)=\left\{\begin{array}{cll}
0 & \text { if } & \chi(t)<c+t \eta(t) b(1-c) \\
{[0,1]} & \text { if } & \chi(t)=c+t \eta(t) b(1-c) \\
1 & \text { if } & \chi(t)>c+t \eta(t) b(1-c)
\end{array}\right.
$$

With a positive bias, the agent is less inclined to intervene than the authority after a sell order: the agent must be more certain that an intervention is needed in order to be willing to deploy it. Thus, if the agent positively weights the noise trader's profit and chooses to intervene following a sale in equilibrium, then the authority benefits. Of course, if the agent's bias is very large, then she will never intervene in equilibrium. We therefore focus on a bias that is not too large, $b<\bar{B} \equiv \min \left\{1 / m_{0}^{*},(J-1) / m_{1}^{*}\right\} .^{27}$

Proposition 7.4 (Biased Interventions). Suppose that $c \in(\widehat{\chi}, 1)$ and that $b \in(0, \bar{B})$. The unique equilibrium of the delegated intervention game is characterized as follows. For $m \in(0,1 / b)$, let

$$
\widetilde{G}(m) \equiv \int_{m}^{\frac{J m}{1-b m}} \frac{t-m}{K_{0} m} f_{s}(t) \mathrm{d} t+\int_{\frac{J m}{1-b m}}^{\infty} \frac{J-1+b t}{K_{0}} f_{s}(t) \mathrm{d} t .
$$

Strategies. The type-0 trader places a sell order distributed according to continuous probability

\footnotetext{
${ }^{26}$ In the appendix, we show that when the agent's bias is not too large, the agent only triggers interventions in equilibrium following a sell order; consequently, we focus on the sell side of the market in the body of the paper.

${ }^{27}$ In addition to ensuring that intervention takes place in equilibrium following a sufficiently large sell order, the bound on the bias also ensures that intervention does not take place following a buy order. In general, intervention following a buy order could help the noise trader: if he buys in state zero, he loses money. By intervening after a buy, the agent makes sure that the noise trader collects one on each share bought for certain. See additional details in the proof of Proposition 7.4.
} 
density function $\phi_{0}(t)$ over support $\left[\widetilde{m}_{0}, \infty\right)$ defined piecewise:

$$
\phi_{0}(t)=\left\{\begin{array}{cl}
\frac{t-\widetilde{m}_{0}}{K_{0} \widetilde{m}_{0}} f_{s}(t) \quad \text { if } \quad t \in\left[\widetilde{m}_{0}, \frac{J \widetilde{m}_{0}}{1-b \widetilde{m}_{0}}\right] \\
\frac{J-1+b t}{K_{0}} f_{s}(t) \quad \text { if } \quad t \in\left[\frac{J \widetilde{m}_{0}}{1-b \widetilde{m}_{0}}, \infty\right)
\end{array}\right.
$$

where $\widetilde{m}_{0}$ is the unique value such that $\widetilde{G}\left(\widetilde{m}_{0}\right)=1$. In addition, $\widetilde{m}_{0} \in\left(m_{0}^{\dagger}, m_{0}^{*}\right)$. The biased agent intervenes with probability zero if $t<J \widetilde{m}_{0} /\left(1-b \widetilde{m}_{0}\right)$ and

$$
\widetilde{\alpha}(t)=1-\frac{J \widetilde{m}_{0}}{t}-b \widetilde{m}_{0} \quad \text { if } \quad t>\frac{J \widetilde{m}_{0}}{1-b \widetilde{m}_{0}} .
$$

Payoffs. The type-0 trader's ex ante expected payoff is $u_{0}=\widetilde{m}_{0}(1-q)$. The authority's expected payoff is strictly greater with delegation $(0<b<\bar{B}))$ than without $(b=0)$.

As in the equilibrium with no bias, the sell orders are broken into a safe zone, in which no intervention occurs, and a risky zone, in which intervention may take place. When the agent is positively biased, she has less incentive than the authority to intervene following sell orders (because interventions harm sellers of the asset). Consequently, the agent must be more convinced than the authority that an intervention is justified. Thus, inside the risky zone, the informed trader can trade more aggressively when the positively biased agent controls the intervention technology, because he is less concerned that his order will induce the agent to intervene: indeed, his mixing density over the risky zone strictly exceeds $\frac{J-1}{K_{0}} f_{s}(t)$, leaving the agent indifferent but benefiting the authority (review (18)). This equilibrium therefore approximates the counter-clockwise rotation of the informed trader's strategy arising in the case of authority commitment. ${ }^{28}$

\section{CONCLUSION}

In this paper we explore a setting in which a privately informed investor trades in an asset in an effort to profit from his knowledge of the underlying state while an authority (a firm manager or government policymaker) who cares intrinsically about the state observes trading activity and decides whether to undertake a costly intervention; e.g., a bailout. We completely characterize the set of perfect Bayesian equilibria of the game and derive a number of results.

We show that there exists a region of the parameter space in which sales orders are partitioned into two sets: a safe zone of small trades that never trigger an intervention and a risky zone of large trades that induce random interventions by the authority in equilibrium. Although the probability of an intervention increases with sell volume over the risky zone, the authority does not expect to benefit from intervening. This occurs because investors employ equilibrium trading strategies that choke off information at the point where the authority is just indifferent about acting. The asset price is non-monotonic in sell volume, falling over the safe zone as the market becomes more

\footnotetext{
${ }^{28}$ The preceding logic applies in reverse to a negatively biased agent, who is more willing than the authority to deploy an intervention which forces the informed trader to pay back on his short position, reducing his profit. Therefore, delegating to an agent with a negative bias strictly reduces the authority's payoff.
} 
convinced that the 'bad' state will obtain and rising over the risky zone as the market becomes more convinced that a corrective intervention will be triggered, resulting in the good state. Indeed, the expected price of the asset is higher in equilibrium than if interventions were not possible. Moreover, to mitigate the probability of an intervention, informed sellers trade less aggressively and trades are, therefore, less Blackwell informative about the underlying state.

Policymakers such as the Federal Reserve frequently advocate using financial markets to inform policy. Our results are less sanguine: the possibility of intervention triggered by sales tend to cause less selling in the first place, reducing the information provided by the market to the point where the policymaker does not benefit. Our results do show that financial markets can inform policy if partial commitment devices, such as delegation or institutional gridlock are present. These devices reduce the probability of an intervention, when the temptation to intervene is strongest.

While the model we present and analyze in this paper is stylized, it identifies key tradeoffs and delivers novel results regarding the use of financial markets to inform policy. A number of interesting, though challenging, avenues remain open for future research. Our model's tractability is enhanced by the assumption that one large strategic trader is present in the market. A natural extension of our analysis could incorporate competition between large, strategic informed investors. Because investors often split their trades into smaller pieces and trade over time, incorporating multiple rounds of trade could generate interesting insights. Also, the ideas and methods used in our analysis could be applied to investigate a variety of related settings; for example, a seller who learns the value of her object by observing bids in an auction and who may decide not to sell if the object is revealed to be highly valuable. Indeed, situations in which strategies of agents both inform and anticipate interventions are ubiquitous, and the question of how policymakers, managers, and other interested parties should act in such settings has never been more relevant.

\section{ACKNOWLEDGEMENTS}

We thank Attila Ambrus, Gennaro Bernile, William Fuchs, Martin Gervais, Itay Goldstein, Brett Green, Miguel Iraola, Kyungmin Kim, Peter Norman Sørensen, and Ming Yang for helpful comments and suggestions. We are grateful to seminar audiences at Berkeley, Drexel, Duke, Emory, Midwest Economic Theory Conference Spring 2014 and 2016, Queen's University, University of Copenhagen, University of Iowa, University of Miami, University of North Carolina, University

of Toronto, and Universite Quebec a Montreal. Taylor gratefully acknowledges support from the National Science Foundation: grant SES-1132187. 


\section{REFERENCES}

Amador, Manuel, and Pierre-Olivier Weill. 2010. "Learning from Prices: Public Communication and Welfare." Journal of Political Economy, 118(5): 866-907.

Angeletos, George-Marios, Guido Lorenzoni, and Alessandro Pavan. 2010. "Beauty Contests and Irrational Exuberance: A Neoclassical Approach." National Bureau of Economic Research Working Paper 15883.

Angeletos, GeorgeMarios, Christian Hellwig, and Alessandro Pavan. 2006. "Signaling in a Global Game: Coordination and Policy Traps." Journal of Political Economy, 114(3): 452-484.

Arrow, Kenneth J., Robert Forsythe, Michael Gorham, Robert Hahn, Robin Hanson, John O. Ledyard, Saul Levmore, Robert Litan, Paul Milgrom, Forrest D. Nelson, George R. Neumann, Marco Ottaviani, Thomas C. Shelling, Robert J. Shiller, Vernon L. Smith, Erik Snowberg, Cass R. Sunstein, Paul C. Tetlock, Philip E. Tetlock, Hal R. Varian, Justin Wolfers, and Eric Zitzewitz. 2008. "The Promise of Prediction Markets." Science, 320: 877-878.

Atakan, Alp E, and Mehmet Ekmekci. 2014. "Auctions, Actions, and the Failure of Information Aggregation." The American Economic Review, 104(7).

Baeriswyl, Romain, and Camille Cornand. 2010. "The signaling role of policy actions." Journal of Monetary Economics, 57(6): 682-695.

Bond, Philip, and Hülya Eraslan. 2010. "Information-based trade." Journal of Economic Theory, 145(5): 1675-1703.

Bond, Philip, and Itay Goldstein. 2014. "Government Intervention and Information Aggregation by Prices." Journal of Finance, forthcoming.

Bond, Philip, Itay Goldstein, and Edward Simpson Prescott. 2010. "Market Based Corrective Actions." Review of Financial Studies, 23(2): 781-820.

Chakravarty, Sugato. 2001. "Stealth-trading: Which traders trades move stock prices?" Journal of Financial Economics, 61(2): 289-307.

Chan, Louis KC, and Josef Lakonishok. 1995. "The behavior of stock prices around institutional trades." The Journal of Finance, 50(4): 1147-1174.

Chen, Qi, Itay Goldstein, and Wei Jiang. 2007. "Price Informativeness and Investment Sensitivity to Stock Price." Review of Financial Studies, 20(3): 619-650.

Chi, Chang-Koo. 2014. "The Value of Information and Posterior Dispersion." University of Wisconsin-Madison Working Paper. 
Cowgill, Bo, and Eric Zitzewitz. 2015. "Corporate Prediction Markets: Evidence from Google, Ford, and Firm X1." Review of Economic Studies, 82(4): 1309-41.

Cowgill, Bo, Justin Wolfers, and Eric Zitzewitz. 2009. "Using Prediction Markets to Track Information Flows: Evidence from Google." University of California Berkeley Working Paper.

Curry, Timothy J., Peter J. Elmer, and Gary S. Fissel. 2003. "Using Market Information to Help Identify Distressed Institutions: A Regulatory Perspective." FDIC Banking Review, 15(10).

Curry, Timothy J, Peter J Elmer, and Gary S Fissel. 2007. "Equity market data, bank failures and market efficiency." Journal of Economics and Business, 59(6): 536-559.

Dow, James, and Gary Gorton. 1997. "Stock Market Efficiency and Economic Efficiency: Is There a Connection?" Journal of Finance, 52(3): 1087-1129.

Dow, James, Itay Goldstein, and Alexander Guembel. 2008. "Incentives for information production in markets where prices affect real investment." University of British Columbia Working Paper.

Easley, David, and Maureen O'Hara. 1987. "Price, trade size, and information in securities markets." Journal of Financial Economics, 19(1): 69-90.

Edmans, Alex, Itay Goldstein, and Wei Jiang. 2014. "Feedback Effects, Asymmetric Trading, and the Limits to Arbitrage." AFA 2013 San Diego Meetings Paper, ECGI - Finance Working Paper No. 318/2011.

Evanoff, Douglas D., and Larry D. Wall. 2002. "Measures of the Riskiness of Banking Organizations: Subordinated Debt Yields, Risk-Based Capital, and Examination Ratings." Journal of Banking and Finance, 989-1009.

Farhi, Emmanuel, and Jean Tirole. 2012. "Collective Moral Hazard, Maturity Mismatch, and Systemic Bailouts." American Economic Review, 102(1): 60-93.

Flannery, Mark J. 1998. "Using Market Information in Prudential Bank Supervision: A Review of the U.S. Empirical Evidence." Journal of Money, Credit and Banking, 30(3): pp. 273-305.

Frame, W. Scott, and Lawrence J. White. 2005. "Fussing and Fuming over Fannie and Freddie: How Much Smoke, How Much Fire?" Journal of Economic Perspectives, 19(2): 159184.

Frankel, Alex, and Navin Kartik. 2016. "What Kind of Central Bank Competence?" Working Paper, Columbia.

Fulghieria, Paolo, and Dmitry Lukin. 2011. "Information Production, Dilution Costs, and Optimal Security Design.” Journal of Financial Economics, 61: 3-42. 
Gandhi, Priyank, and Hanno Lustig. 2010. "Size Anomalies in U.S. Bank Stock Returns: a Fiscal Explanation.” NBER Working Paper No. 16553.

Glosten, Lawrence R., and Paul R. Milgrom. 1985. "Bid, ask and transaction prices in a specialist market with heterogeneously informed traders." Journal of Financial Economics, 14(1): $71-100$.

Goldstein, Itay, Emre Ozdenoren, and Kathy Yuan. 2011. "Learning and Complementarities in Speculative Attacks." Review of Economic Studies, 78: 263-292.

Grossman, Sanford J., and Joseph E. Stiglitz. 1980. "On the Impossibility of Informationally Efficient Markets." American Economic Review, 70(3).

Hanson, Robin, and Ryan Oprea. 2009. "A Manipulator Can Aid Prediction Market Accuracy." Economica, 76(302): 304-314.

Hendershott, Terrence, Dmitry Livdan, and Norman Schürhoff. 2015. "Are Institutions Informed About News?" Journal of Financial Economics, 117(2): 249-87.

Jewitt, Ian. 2007. "Information order in decision and agency problems." University of Oxford Working Paper.

Kau, James B, James S Linck, and Paul H Rubin. 2008. "Do managers listen to the market?" Journal of Corporate Finance, 14(4): 347-362.

Ke, Bin, Steven Huddart, and Kathy Petroni. 2003. "What insiders know about future earnings and how they use it: Evidence from insider trades." Journal of Accounting and Economics, 35(3): 315-346.

Kurlat, Pablo, and Laura Veldkamp. 2015. "Should We Regulate Financial Information?" Journal of Economic Theory, 158: 697-720.

Kyle, Albert S. 1985. "Continuous Auctions and Insider Trading." Econometrica, 53(6): 13151335.

Lehmann, EL. 1988. "Comparing Location Experiments." The Annals of Statistics, 521-533.

Luo, Yuanzhi. 2005. "Do Insiders Learn from Outsiders? Evidence from Mergers and Acquisitions." The Journal of Finance, 60(4): 1951-1982.

Malkiel, Burton G., and Eugene F. Fama. 1970. "Efficient Capital Markets: A Review Of Theory And Empirical Work." The Journal of Finance, 25(2): 383-417.

Mitchell, Wesley, and Arthur Burns. 1938. "Statistical Indicators of Cyclical Revivals." In Business Cycle Indicators. , ed. Geoffrey Moore. Princeton University Press. NBER Bulletin 69, New York. 
O'Hara, Maureen, and Wayne Shaw. 1990. "Deposit Insurance and Wealth Effects: The Value of Being "Too Big to Fail”." The Journal of Finance, 45(5): 1587-1600.

Ottaviani, Marco, and Peter Norman Sørensen. 2007. "Outcome manipulation in corporate prediction markets." Journal of the European Economic Association, 5.2(3): 554-563.

Philippon, Thomas, and Vasiliki Skreta. 2010. "Optimal Interventions in Markets with Adverse Selection.” NBER Working Paper No. 15785.

SIGTARP. 2011. "Extraordinary Assistance Provided to Citigroup, Inc." http://online.wsj.com/public/resources/documents/CitiOIG.pdf.

Snowberg, Erik, Justin Wolfers, and Eric Zitzewitz. 2012. "Prediction Markets for Economic Forecasting." NBER Working Paper No. 18222.

Stock, James H., and Mark W. Watson. 2003. "Forecasting Output and Inflation: The Role of Asset Prices." The Journal of Economic Literature, 41(3): 788-829.

Subrahmanyam, Avanidhar, and Sheridan Titman. 1999. "The Going-Public Decision and the Development of Financial Markets." Journal of Finance, 54(3): 1045-1082.

Tirole, Jean. 2012. "Overcoming Adverse Selection: How Public Intervention Can Restore Market Functioning." American Economic Review, 102(1): 29-59.

Wolfers, Justin, and Eric Zitzewitz. 2004. "Prediction Markets." Journal of Economic Perspectives, 18(2): 107-126. 